\title{
Robust Adaptive Neural Network Control for PEM Fuel Cell
}

\author{
Alireza Abbaspour ${ }^{1}$, Arash Khalilnejad ${ }^{2}$, Zheng Chen*3 \\ 1-Florida International University, Miami, Florida, United States \\ 2-Case Western Reserve University,Cleveland, Ohio, United States \\ 3-Wichita State University, Wichita, Kansas, United States
}

\begin{abstract}
This paper presents a robust neural network adaptive control for polymer electrolyte membrane (PEM) fuel cells (FCs). Since deviations between the partial pressure of hydrogen and oxygen in PEMFCs lead to serious membrane damage, it is desirable to have a robust and adaptive control to stabilize the partial pressure, which can significantly lengthen their lifetime. Due to inherent nonlinearities in PEMFC dynamics and variations of the system parameters, a linear control with fixed gains cannot control the PEMFC system properly. Therefore, a neural network adaptive control with feedback linearization is developed for this system. With a feedback linearization control only, the performance is deviated in the presence of unknown dynamics and disturbances. Thus, a robust adaptive neural network control is added to the feedback linearization control to reduce the deviation. Simulation results show that the proposed control can significantly enhance the output performance as well as reject the disturbances.
\end{abstract}

Keywords: Adaptive control, PEMFC, Nonlinear dynamic, Neural Network, Robustness

\section{INTRODUCTION}

Environmental friendly energy sources received great attention in recent decades. Fuel cells (FCs) are one of these environmental friendly energy devices that produce electricity, heat, and water through a chemical reaction [1]. Being remarkably energy efficient, emitting small amount of the sulfur oxide and nitrogen oxide, and absolutely low noise producing, are the main factors that make FCs as one of the best clean source of energy.

Damage to the fuel cell membranes can cause destructive degradation in the stack voltage and oxygen exhaustion. An accurate air and hydrogen flow rate control can helps to keep the Hydrogen and Oxygen pressure a desired fixed amount in the fuel cell system and

*Corresponding author: Zheng Chen

Email Address: zheng.chen@wichita.edu 
prevents membrane damage [1]. Due to the nonlinear properties of chemical reactions, linear control strategies cannot control fuel cells efficiently. Therefore, nonlinear model based control strategies are potential candidates for these kinds of highly nonlinear systems [2]. These kinds of controllers need an accurate mathematical model in their design. Several dynamic models of the polymer electrolyte membrane fuel cell (PEMFC) were introduced [1-9]. Purkrushpan et al. introduced a mathematical model for PEMFC control application [1]. This model contains the flow characteristics, compressor dynamic, manifold (anode and cathode), membrane humidity, and reactant partial pressures. Nonlinearities and uncertainties inherent in the load and stack voltage, as well as the state equations make the nonlinear control development very challenging [1,5]. A nonlinear dynamic model for gas pressure control was introduced by $\mathrm{Na}$ and Gou [2]. This model was applicable to control the pressure using nonlinear control strategies., i.e., feedback linearization control technique.

Restrepo et al. used parameter identification to introduce an equivalent circuit based model of PEMFC. In this model two circuit were considered where, one of them described the output voltage characteristics and the other one described the thermal characteristics [8]. The parameter identification was done using an evolutionary technique. In another approach, nonlinear autoregressive with exogenous input (NARX) and nonlinear output error (NOE) neural network structure was used to obtain a time varying model of the stack voltage in PEMFC which was applicable to predictive control system design [9].

Adaptive and robust control of the PEMFC is a major concern for many researchers and received an increasing attention among them [10-19]. Almeida et al. introduced an optimal artificial neural network (ANN) based control for the PEMFC in order to obtain an approximated optimal control [10]. Rezazadeh et al. designed a radial basis function neural network (RBFNN) adaptive inverse controller for the PEMFC system to control the voltage output [11]. This control scheme, which is a combination of proportional derivative (PD) and RBFNN, did not require any parameter identification of the system. Therefore, it can reduce the needed experimental data for the control design. A gain-scheduling-based adaptive controller was designed for PEM by Farcas and Dobra [12]. This adaptive controller was used to obtain a certain control performance for the management of the membrane conductivity. This controller was implemented with a 2 proportional-integral-derivative (PID) structure. Abbaspour et al. optimized the performance of the PEMFCs feedback linearization controller via a genetic algorithm [13]. The optimization process was performed on the linear gains of the designed controller by the non-dominated sorting genetic algorithm II (NSGA-II) to obtain an accurate controller.

Beirami et al. tried to optimize the oxygen excess ratio to obtain the maximum power tracking [14]. In their design, a feedforward fuzzy logic controller with PID feedback was used, and the controller parameters were optimized with a self-adaptive differential algorithm at the same time. Ou et al. also introduced a feedforward fuzzy PID (FFPID) algorithm to control the oxygen pressure ratio [15]. This adaptive controller tunes the PID control gains through on-line fuzzy logic optimization loop to regulate air flow rate. In another design, 
Benchouia et al. introduced an adaptive fuzzy logic controller for stack voltage control of the PEMFC [16]. Despite the advantages of the fuzzy controllers in being robust against uncertainties, their performance is inaccurate in comparison with model based control strategies; thus, they are more suitable for the systems that designer does not have access to an accurate dynamic model.

Wang et al. investigated the robustness of an $\mathrm{H}^{\circ} \infty$ controller of the PEMFC on several platforms and concluded that this controller can be implemented on different modules without any modification[17]. However, it is important to mention that the resulting controller is only optimal in the predefined cost function and its performance in other aspects is not guarantied. A fault tolerant control design for PEMFC was introduced by Li et al. [18]. In this design, fisher discriminant analysis (FDA) and support vector machine (SVM) techniques were used to detect and isolate the faults in the components of the PEMFC system, thus, partial failure of sensors and actuators cannot degrade the system performance.

In this paper, a robust adaptive neural network control with feedback linearization is developed for the PEMFC to stabilize the pressure of hydrogen and oxygen. The nonlinear model of the PEMFC and the feedback linearization controller to cancel the nonlinearity in the model and stabilize the hydrogen and oxygen partial pressure was developed by $\mathrm{Na}$ and Gou [2]. However, since the feedback linearization control is vulnerable in the presence of unknown dynamics, and uncertainties and the gains of the linear stabilizing control are sensitive to the output performance [12], this paper introduces a robust adaptive neural network to overcome the above issues. An adaptive neural network, which is simple in its structure, is designed for the feedback linearization control to compensate for system uncertainties and disturbances as well as optimize the stabilizing control to improve the PEMFC output performance. This adaptive controller is capable of on-line adaptive learning so that it can adjust to variations and disturbances in system parameters. As a result, the amount of required modeling information can be reduced significantly. This means that with minimum modifications, this controller can be applied for different modules of the PEMFC and the controller does not need exact details of the model. Nonetheless, by using the adaptive neural network controller, the need for tuning linear gains in the feedback linearization controller is eliminated. Simulation results have shown that this robust and adaptive design not only improved the accuracy of the controller, but also improved the robustness of the PEMFC against disturbances and uncertainties.

The paper is organized as follows: Section 2 provides the PEMFC model specification, while the proposed control design is illustrated in Section 3. Then, in Section 4, the numerical simulation results are presented to demonstrate the effectiveness of the introduced method. Finally, the conclusions and future work are provided in Section 5. 


\section{PEMFC DYNAMIC MODEL}

PEMFCs are made up of an electrolyte membrane which is placed in the middle of two electrodes (cathode and anode). Only the electrolyte ions are allowed to exit, but not the electrons. Under the potential difference between the cathode and anode, the electricity can be produced by flowing electrons over an external circuit. The electricity production of PEM fuel cells is the outcome of an electrochemical reaction between the hydrogen gas (anode) and oxygen gas (cathode). This chemical reaction produces electricity, water, and heat.

Fig. 1 shows the PEMFC components. As shown, hydrogen gas enters from the anode gas diffusion layer and meets the anode catalyst layer. Then, the catalyst helps the hydrogen proton $H^{+}$to separate its electrons and moves through the proton exchange membrane to the other side. At the same time, the electrons pass through a load toward the cathode plate where they meet the cathode gas diffusion layer. On the other side, the oxygen molecules are separated into distinct oxygen atoms in the presence of a catalyst on the cathode side. Subsequently, a combination reaction occurs. Finally, an $\mathrm{H}_{2} \mathrm{O}$ water molecule, electricity, and heat are obtained in the reaction between one oxygen atom and two $H^{+}$protons.

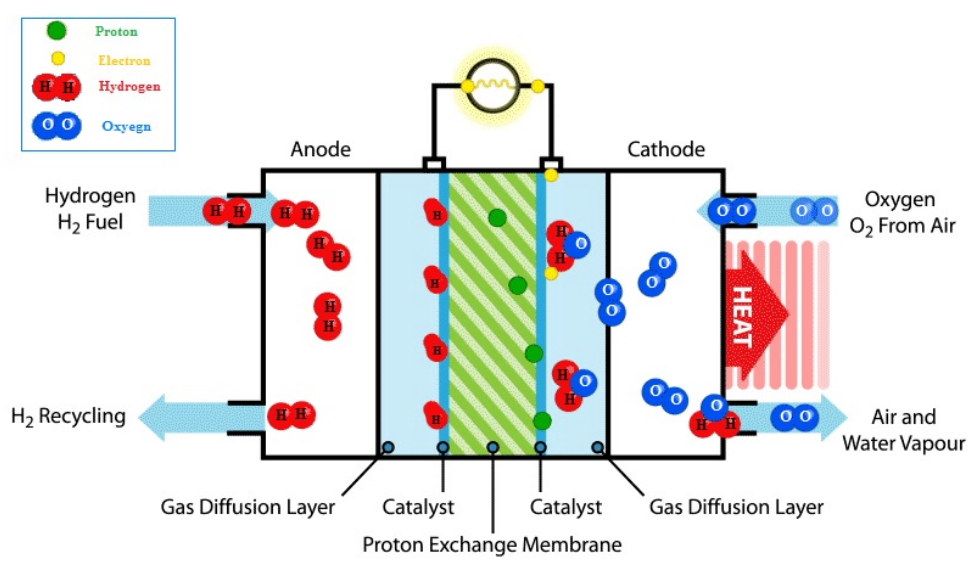

Figure 1: PEM Fuel Cell operation diagram [12].

Hydrogen can be produced by a fuel processor on the anode side which is called reformer. This reformer can be replaced by hydrogen pressurized gas capsule. In order to use the reformer as a hydrogen producer, a regulator for the pressure and a purifier for obtaining purified hydrogen are needed as well. On the cathode side, the pressure of oxygen gas must also be controlled. Thus, an air supplier system that consists of an air flow controller, air filter, and compressor is needed to have a controlled oxygen pressure. Dehydration of the fuel cell membrane should be avoided; therefore, a humidifier is needed on both sides (anode and cathode). Moreover, a water container, a pump, a water filter, and a heat exchanger might be required for the heat and water level control in the fuel cell systems [1, 20,21]. According to the polarization I-V curve (Fig. 2), each cell can produce voltage between 0 and $1 \mathrm{~V}[20,21]$. Therefore, in order to produce higher voltage, several cells can be connected 
in series. As shown here, the I-V curve has a nonlinear relationship, mainly depended on cell temperature, current density, reactant pressure, and membrane humidity.

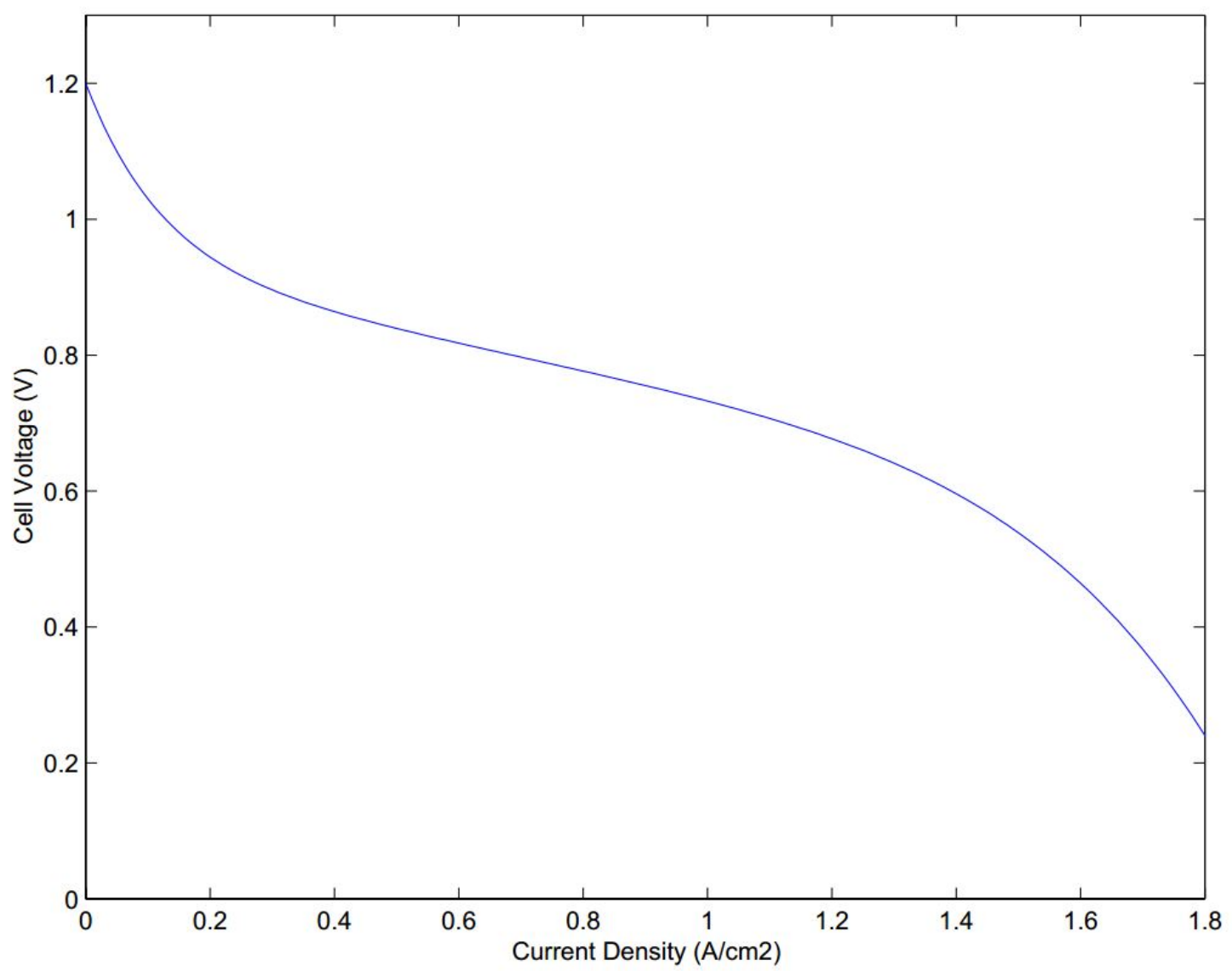

Figure 2: PEMFC polarization curve at 70C [20].

Here, the stack voltage $V_{s t}[20]$ can be defined as:

$$
V_{\text {st }}=E-V_{\text {activation }}-V_{\text {ohmic }}-V_{\text {concentration }}
$$

where $E$ is the cell thermodynamic potential, $V_{\text {activation }}$ is voltage which is lost because of the reaction rate on the electrode surface, $V_{\text {ohmic }}$ is ohmic voltage loss caused by the proton flow resistance in the electrolyte, and $V_{\text {concentration }}$ is voltage drop due to the decline in gas pressure or the mass of hydrogen and oxygen transportation. These variables can be defined with following equations [20]:

$$
\begin{gathered}
E=N_{0}\left[V_{o}+(R T / 2 F) \ln \left(P_{H_{2}} \sqrt{P_{O_{2}}} / P_{H_{2} O_{c}}\right)\right] \\
V_{\text {activation }}=N \frac{R T}{2 \alpha F} \ln \left(\frac{I_{f c}+I_{n}}{I_{0}}\right) \\
V_{\text {ohm }}=N I_{f c} r
\end{gathered}
$$




$$
V_{\text {concentration }}=N \operatorname{mexp}\left(n I_{f c}\right)
$$

where $P_{\mathrm{O}_{2}}$ and $P_{\mathrm{H}_{2}}$ are the oxygen and hydrogen pressures, respectively, and $P_{\mathrm{H}_{2} \mathrm{O}_{c}}$ is the water pressure on the cathode side. $N$ is the number of cells in the FC system, $V_{o}$ is the open circuit voltage of cell, $R$ is the universal gas constant, $T$ is the FC temperature, $F$ is Faraday constant, $\alpha$ is the charge transfer coefficient, $I_{f c}$ is the density of output current, $I_{o}$ is the exchange current density, $I_{n}$ is the internal current density, $m$ and $n$ are constants in mass transfer voltage, and $r$ is the resistant related to the area coefficient.

A dynamic mathematical model of PEMFC is obtained from [2]. Dynamics on anode side are governed by the following equations:

$$
\begin{gathered}
\frac{d P_{H_{2}}}{d t}=\frac{R T}{V_{a}}\left[u_{a} k_{a} Y_{H_{2}} \lambda_{H_{2}}-C_{1} I_{f c}-\left(u_{a} k_{a} \lambda_{H_{2}}-C_{1} I_{f c}\right) F_{H_{2}}\right] \\
\frac{d P_{H_{2} O_{a}}}{d t}=\frac{R T}{V_{a}}\left[u_{a} k_{a} \lambda_{H_{2}} \frac{\phi_{a} P_{v s}}{P_{H_{2}}+P_{H_{2} O_{a}}-\phi_{a} P_{v s}}-\left(u_{a} k_{a} \lambda_{H_{2}}-C_{2} I_{f c}\right) F_{H_{2} O_{a}}-C_{2} I_{f c}\right]
\end{gathered}
$$

Dynamics related to the cathode are governed by the following equations:

$$
\begin{gathered}
\frac{d P_{O_{2}}}{d t}=\frac{R T}{V_{c}}\left[u_{c} k_{c} Y_{O_{2}} \lambda_{O_{2}}-\frac{C_{1}}{2} I_{f c}-\left(u_{c} k_{c} Y_{N_{2}} \lambda_{O_{2}}-\frac{C_{1}}{2} I_{f c}\right) F_{O_{2}}\right] \\
\frac{d P_{N_{2}}}{d t}=\frac{R T}{V_{c}}\left[u_{c} k_{c} Y_{N_{2}} \lambda_{O_{2}}-u_{c} k_{c} \lambda_{O_{2}} F_{N_{2}}\right] \\
\frac{d P_{H_{2} O_{2}}}{d t}=\frac{R T}{V_{c}}\left[\begin{array}{c}
u_{c} k_{c} \lambda_{O_{2}} \frac{\phi_{c} P_{v s}}{\left(u_{c} k_{c} \lambda_{O_{2}}+C_{1} I_{f c}+C_{N_{2}}+P_{H_{2} O_{c}-\phi_{c} P_{v s}} I_{f c}\right) C_{H_{2} O_{c}}+C_{2} I_{f c}-} \\
P_{f c}
\end{array}\right]
\end{gathered}
$$

where the anode variables are denoted with a subscript $a$; the cathode variables are denoted with a subscript $c ; u_{a}$ and $u_{c}$ are the flow rates of hydrogen and oxygen gases ,respectively , which are used as control input in the proposed system; $k_{a}$ and $k_{c}$ are conversion factors; $Y_{\mathrm{H}_{2}}, Y_{\mathrm{O}_{2}}$, and $Y_{\mathrm{N}_{2}}$ are the hydrogen, oxygen, and nitrogen initial mole fractions, which are set to be $0.99,0.21$, and 0.79 , respectively; $\lambda_{O_{2}}$ is the ratio of the oxygen supplied to the cathode; $\lambda_{H_{2}}$ is the ratio of hydrogen supplied to the anode; $\phi_{a}$ and $\phi_{c}$ are the associated humidity on the cathode and anode sides, respectively; $P_{v s}$ is the saturation pressure, which can be obtained from thermodynamics tables; $F_{\mathrm{H}_{2}}, F_{\mathrm{H}_{2} \mathrm{O}_{a}}, F_{\mathrm{O}_{2}}, F_{\mathrm{N}_{2}}$, and $F_{\mathrm{H}_{2} \mathrm{O}_{c}}$ are the fractions that are defined to simplify the equations (6-10) [2]:

$$
\begin{aligned}
& F_{O_{2}}=\frac{P_{O_{2}}}{P_{O_{2}}+P_{N_{2}}+P_{H_{2} O_{c}}}, F_{H_{2}}=\frac{P_{H_{2}}}{P_{H_{2}}+P_{H_{2} O_{a}}}, \\
& F_{N_{2}}=\frac{P_{N_{2}}}{P_{O_{2}}+P_{N_{2}}+P_{H_{2} O_{c}}}, F_{H_{2} O_{a}}=\frac{P_{H_{2} O a}}{P_{H_{2}}+P_{H_{2} O_{a}}} \\
& F_{H_{2} O_{c}}=\frac{P_{H_{2} O_{c}}}{P_{O_{2}}+P_{N_{2}}+P_{H_{2} O_{c}}}
\end{aligned}
$$

\section{CONTROL DESIGN}

In this section, the control system proposed for the PEMFC system is introduced and illustrated. 


\subsection{Feedback Linearization Control Technique}

Since the proposed control structure is mainly based on the feedback linearization control technique, the basic principles of the feedback linearization controller are provided here to clarify the control design. In this technique, by using feedback control signal the inherent nonlinear dynamic will be removed and concurrently a desired linear system response will be achieved [22]. In order to illustrate the structure of the feedback linearization method, consider an $n t h$ order and affine nonlinear system with $m$ input represented by:

$$
\begin{aligned}
& \dot{x}=f(x)+G(x) u, \\
& y=h(x)
\end{aligned}
$$

where the system can be separated based on their dependency on control inputs with $f($. and $G(.) . f($.$) is the matrix function related to variables that are depended on control input$ $(u)$, and $G$ is an $n \times m$ control effectiveness matrix function that is related to $u$. To obtain such affine relation (12), consecutive differentiations of system output should be done until a linear relationship between the control input and the system output appears [22]. Now by assuming $h(x)=x$, in the first derivative of the output y a clear affine relation for $\mathrm{u}$ is obtained.

$$
\dot{y}=\dot{x}=f(x)+G(x) u
$$

This linear relation can be applied (if $G(x) \neq 0$ ) by choosing

$$
u=G(x)^{-1}[\dot{x}-f(x)]
$$

Finally, by replacing the system dynamics with the desired one, yields

$$
u=G(x)^{-1}\left[\dot{x_{d}}-f(x)\right]
$$

where the subscript $d$ denotes the desired dynamic. These desired dynamics can be obtained by linear controllers, such as PD, PI, or PID.

\subsection{PEMFC Feedback Linearization Control Design}

This section explains the feedback linearization control design for PEMFC air supply developed by $\mathrm{Na}$ [2]. The feedback linearization control design for PEMFC air supply is obtained from the model described by (6-10). In this study, the feedback linearization controller is designed to control the oxygen and hydrogen pressures. In this design, the stack current is considered as a disturbance to the PEMFC system [6]. The nonlinear affine dynamic in presence of disturbances can be presented as:

$$
\begin{aligned}
& \dot{x}=f(x)+g(x) u+p(x) d \\
& y=h_{(x),}
\end{aligned}
$$

where $x$ is the state vector, $\mathrm{u}$ the input or control vector, and $f(x)$ and $g(x)$ are the functions that separates state parameters related to $u$. The $d$ denotes the disturbance variables; $p(x)$ is 
the dimensional vector field of the disturbance, and $h(x)$ is the system output. Regarding (610), the specified outputs and the disturbance, and dividing the control inputs, the parameter of PEMFC nonlinear model involved in the air supply system presented as

$$
\begin{array}{r}
g(x)=\left[\begin{array}{cc}
\frac{R T \lambda_{H_{2}}}{V_{a}}\left(k_{a} Y_{H_{2}}-k_{a} F_{H_{2}}\right) & 0 \\
0 & \frac{R T \lambda_{O_{2}}}{V_{c}}\left(k_{c}\right) Y_{O_{2}}-k_{c} Y_{N_{2}} F_{O_{2}}
\end{array}\right], \\
X=\left[\begin{array}{c}
P_{H_{2}} \\
P_{H_{2} O_{a}} \\
P_{O_{2}} \\
P_{N_{2}} \\
P_{H_{2} O_{c}}
\end{array}\right], U=\left[\begin{array}{l}
u_{a} \\
u_{c}
\end{array}\right], Y=\left[\begin{array}{l}
-\frac{C_{1}}{V_{a}}+\frac{C_{1}}{V_{a}} F_{H_{2}} \\
P_{O_{2}}
\end{array}\right], p(x)=R T\left[\begin{array}{c}
C_{1} \\
-\frac{C_{1}}{2 V_{c}}+\frac{C_{c}}{2 V_{c}} F_{O_{2}}
\end{array}\right] \\
d=I_{f c}, f(x)=0
\end{array}
$$

Now, the nonlinear control law for the multiple-input-multiple-output (MIMO) nonlinear PEMFC system can be extracted from (17). The disturbance in (17) can be used in the control design, if there is a condition to measure the disturbance. Since the output current $I_{f c}$ is measurable, the mentioned condition is satisfied. Therefore, the feedback linearization control law can be obtained as

$$
u=g^{-1}(x)\left(\left[\begin{array}{c}
\dot{P}_{H_{2 D}} \\
\dot{P}_{O_{2 D}}
\end{array}\right]-p(x) d\right)
$$

Considering that $\dot{y_{1}}=\dot{P_{H_{2}}}$ and $\dot{y_{2}}=\dot{P_{O_{2}}}$, in order to guarantee that $P_{H_{2}}$ and $P_{O_{2}}$ are settled to the desired values (in atmosphere) of $P_{\mathrm{H}_{2} \mathrm{D}}$ and $P_{\mathrm{O}_{2} \mathrm{D}}$, a stabilizing linear controller is required.

\subsection{Stabilizing Linear Controller}

In this subsection, linear stabilizing control for PEMFC developed by $\mathrm{Na}$ [2] is introduced. The stabilizing controller design is based on the pole-placement technique [23]. This section provides the pole-placement principle of designing a stabilizing controller. The new control inputs are given by

$$
\left[\begin{array}{l}
v_{1} \\
v_{2}
\end{array}\right]=\left[\begin{array}{c}
\dot{y}_{1 d}-k_{11} e_{1} \\
\dot{y}_{2 d}-k 21 e_{2}
\end{array}\right]
$$

where $e_{1}=y_{1}-y_{1 d}$, and $e_{2}=y_{2}-y_{2 d}$.

Despite the fact that the nonlinear PEMFC system is precisely linearized by feedback linearization, variation of the parameters can cause degradation in the control performance, particularly when the load varies. Thus, to avoid this degradation, (19) is modified with adding two integral in the closed-loop system [2, 24]:

$$
\left[\begin{array}{l}
v_{1} \\
v_{2}
\end{array}\right]=\left[\begin{array}{l}
\dot{y}_{1 d}-k_{11} e_{1}-k_{12} \int e_{1} d t \\
\dot{y}_{2 d}-k 21 e_{2}-k_{22} \int e_{2} d t
\end{array}\right]
$$


From (19), the error dynamics can be extracted:

$$
\begin{array}{r}
\ddot{e}_{1}+k_{11} \dot{e}_{1}+k_{12} e_{1}=0 \\
\ddot{e}_{2}+k_{21} \dot{e_{2}}+k_{22} e_{2}=0
\end{array}
$$

The stabilizing controller can be designed by properly selecting the roots of ' $s$ ' in $s^{2}+k_{11} s+$ $k_{12}$ and $s^{2}+k_{12} s+k_{22}$. Furthermore, the overshoots can be minimized by considering the following conditions: $k_{11}^{2} \geq 4 k_{12}$ and $k_{21}^{2} \geq 4 k_{22}[23,24]$.

\subsection{Adaptive Neural Network Design}

The feedback linearization control method suffers from inaccuracy in the system modeling and uncertainties. Here, a mathematical proof is provided to demonstrate that this method is vulnerable against un-modeled dynamics and uncertainties. Consider (12) with the uncertainties term $(\Delta f, \Delta G))$.

$$
\dot{x}=f(x)+\Delta f(x)+(G(x)+\Delta G(x)) u
$$

Assuming that this system describes the actual system, by applying feedback linearization to it, the uncertainties parts are unknown to the controller $\left(u=G^{-1}(x)(v-f(x)), \dot{x}=v\right)$, and by applying this input to the system we have:

$$
\begin{array}{r}
\dot{x}=f(x)+\Delta f(x)+(G(x)+\Delta G(x)) G^{-1}(x)(v-f(x))= \\
\Delta f(x)-\Delta G(x) G^{-1}(x) f(x)+\left(I_{n \times n}+\Delta G(x) G^{-1}(x)\right) v
\end{array}
$$

where $I_{n \times n}$ is an $n \times n$ identity matrix. As can be seen, the linear relation of $(\dot{x}=v)$ can only be obtained when $\Delta f(x)=\Delta G(x)=0$. Otherwise, in the presence of uncertainties, the system can no longer be considered linear, and this may degrade the feedback linearization control performance. To tackle this problem, this paper suggests using an adaptive neural network $(\mathrm{NN})$ in the feedback linearization structure. Basically, it is demonstrated that an $\mathrm{NN}$ is capable of approximating extremely nonlinear and uncertain systems within the needed precision [23]. In this section, an online learning $\mathrm{NN}$ is used as an adaptive control element $\left(u_{a d}\right)$ in the feedback linearization control. This NN structure has been used for designing flight controller in several research studies [26-28]. Here, in this paper we designed a three layer neural network because of its computational feasibility and its simplicity for the application. The proposed NN structure is developed to estimate the dynamic model error of PEMFC system.

A three layer adaptive NN signal can be presented by [25].

$$
u_{a d}=\hat{W} \sigma\left(\hat{V}^{T} \mu\right)
$$

where $V$ and $W$ are the weight matrices in the first and third layer, respectively. As Fig. 3 shows, this two weighting matrices join the first and the third layer together. $\sigma$ is the activation function, and $\mu$ is the neural network input [25].

$$
\dot{\hat{W}}=-\Gamma_{W}\left[\left(\hat{\sigma}-\sigma^{\prime} \hat{V}^{T} \mu\right) e^{T} P+k_{e}\|e\| \hat{V}\right]
$$




$$
\begin{gathered}
\dot{\hat{V}}=-\Gamma_{V}\left[\mu e^{T} P W^{T} \text { sigma }^{\prime}+k_{e}\|e\| \hat{V}\right] \\
\sigma(z)=\left[1, \sigma_{1}\left(z_{1}\right), \ldots, \sigma_{n_{2}}\left(z_{n_{2}}\right)\right]^{T} \in R^{n_{2}+1} \\
\sigma(z)=\left[1, x^{T}\right]^{T}
\end{gathered}
$$

where $\Gamma_{W}$ and $\Gamma_{V}$ are the learning coefficient gains, $e$ is the model dynamic error, and $P$ is a diagonal positive definite matrix that can be extracted from the Lyapunov function [22]:

$$
A^{T} P+P A=-Q
$$

where $A$ is Hurwitz and for any $Q>0$, a unique $P>0$ exists. $\sigma^{\prime}$ is the partial derivative of $\sigma$ with respect to $z=\hat{V}^{T} \mu$, and the scalar functions $\sigma_{i}$ are sigmoid activation functions that represent the "firing" characteristics of the neuron, as follows:

$$
\begin{gathered}
\hat{\sigma}^{\prime}=\left.\frac{d \sigma}{d z}\right|_{z=\hat{V}^{T} \mu}=\left[\begin{array}{ccc}
0 & \ldots & 0 \\
\frac{d \sigma_{1}}{d z_{1}} & \ldots & 0 \\
\vdots & \ddots & \vdots \\
0 & \ldots & \frac{d \sigma_{n 2}}{d z_{n 2}}
\end{array}\right] \in R^{\left(n_{2}+1\right) \times n_{1}} \\
\sigma_{i}\left(z_{i}\right)=\frac{1}{1+e^{-a_{i} z_{i}}}, i=1,2, \ldots, n_{2} .
\end{gathered}
$$

where $a_{i}$ are activation potential factors and they should be normally distinct values for each neuron. The structure of NN can be seen in Fig. 3. In this NN structure we have two inputs and one output where the input of $\mathrm{NN}$ are the value of the controlled parameter and the error of control parameter. The output of neural network is a control signal which is added to the feedback signal to compensate for the nonlinearities and improve the control accuracy.

\section{NUMERICAL SIMULATION}

In this paper, a robust neural network adaptive controller is introduced to overcome problems associated with the limited knowledge of the PEMFC dynamic model. Fig. 4 presents an overall view of the designed controller, this controller is consisted of a feedback linearization controller, two PI controller to tuned the feedback linearized system, and two NN structure to adapt the system with nonlinearities, uncertainties and disturbances As previously mentioned, the feedback linearization control is vulnerable when facing disturbances and uncertainties. Thus, three scenarios are considered to evaluate the performance of proposed controller: 1) The controller is examined in a normal condition. 2) We introduced an immeasurable disturbance into the oxygen and hydrogen partial pressure (Fig. 10) of the system to examine our proposed design. 3) Uncertainties in hydrogen and oxygen pressure are added to system for checking the controller robustness. All these three scenarios are done in three controllers: The proposed adaptive controller, the feedback linearization controller, and the linear proportional integral (PI) controller which are denoted in the figures with NN adaptive control, nonlinear control, and linear control, respectively. Since the 


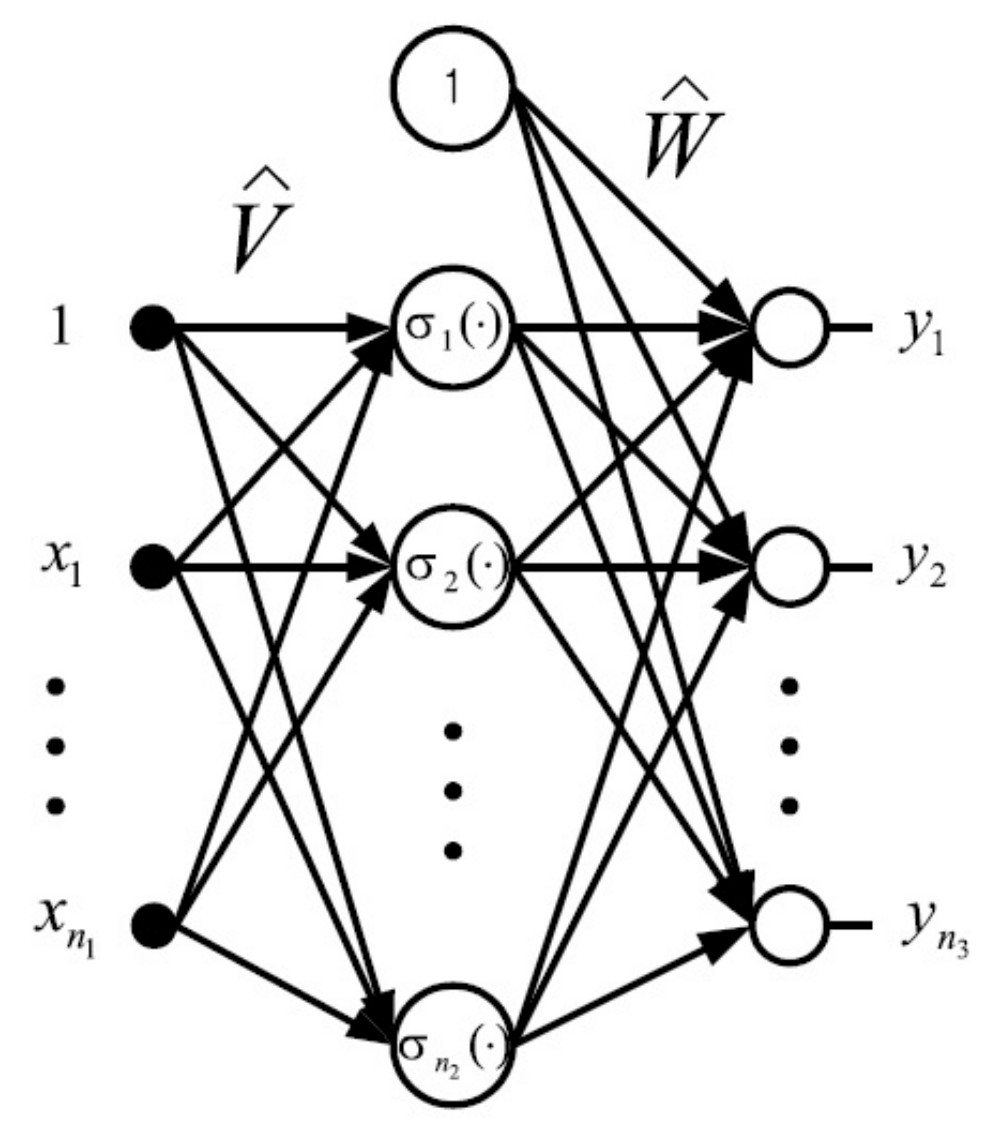

Figure 3: Structure of three-layer NN [25].

disturbances and uncertainties were immeasurable, the feedback linearization control did not use any information from the disturbance. In this section, the results of simulations are provided to demonstrate the advantages of proposed design. The objective in this design is to control the hydrogen and oxygen partial pressure with desired values ( 3 atmospheres). Parameters in the stabilizer controller (26) were selected as $k_{12}=k_{22}=5$ and $k_{11}=k_{21}$ to establish the mentioned condition, while the NN learning coefficient gains were tuned by genetic algorithm to obtain the optimal performance [28]:

$\Gamma_{w 1}=0.354, \Gamma_{v 1}=4.998, \Gamma_{w 2}=0.08215, \Gamma_{v 2}=0.1962$.

Load profile details, which were used for evaluation of the controller, are depicted in Fig. 5, where the load resistances were varied from 0.145 to $4.123 \Omega$ during the simulation period, which introduced a measurable disturbance in $I_{f c}$. In this simulation, the stack temperature was assumed to be constant at $353 \mathrm{~K}$. Then the numerical simulations are done using using a core i7 desktop computer with MATLAB SIMULINK software.. The characteristics of the PEMFC system which is used in this paper is shown on Table.1. 


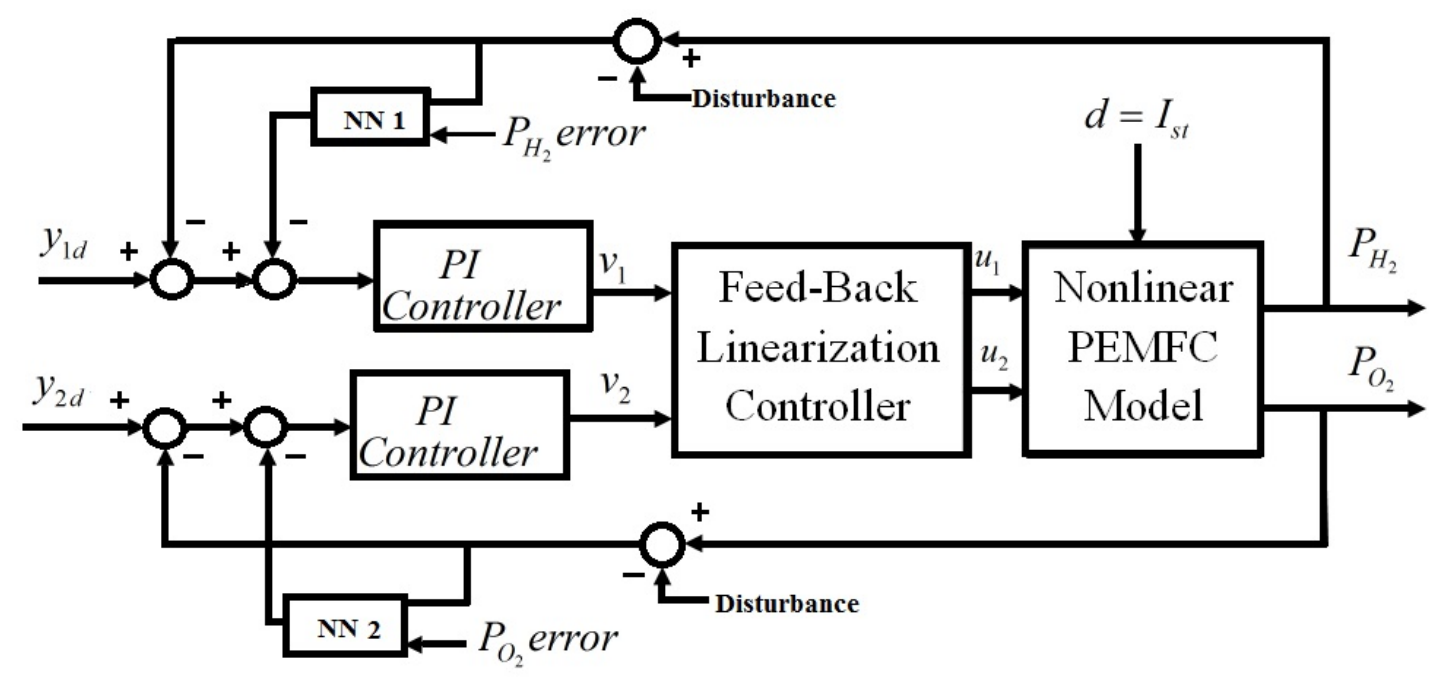

Figure 4: Overview of the designed controller.

Table 1: PEMFC Parameters.

\begin{tabular}{|l|l|l|}
\hline Parameter & Value & Description \\
\hline$R$ & 8.314 & $\begin{array}{l}\text { Universal gas constant } \\
{[\mathrm{J} / \text { mol-k] }}\end{array}$ \\
\hline$F$ & 96485 & Faraday constant $[\mathrm{C} / \mathrm{mole}]$ \\
\hline$T$ & 353 & Fuel cell temperature $[\mathrm{K}]$ \\
\hline$V_{0}$ & 1.028 & Open cell voltage \\
\hline$N$ & 30 & Cell number \\
\hline$I_{x z}$ & 0.75 & $X Z$ Plane Inertia Moment \\
\hline$\alpha$ & 0.5 & $\begin{array}{l}\text { Coefficient of charge trans- } \\
\text { fer }\end{array}$ \\
\hline$m$ & $2.11 \times 10^{-5}$ & Mass transfer voltage $[\mathrm{V}]$ \\
\hline$n$ & $2.45 \times 10^{-3}$ & $\begin{array}{l}\text { Mass transfer } \\
{\left[\mathrm{cm}^{2} \mathrm{~m}^{-1}\right]}\end{array}$ \\
\hline$r$ & $2.45 \times 10^{-4}$ & $\begin{array}{l}\text { electrical } \\
{\left[k \Omega \mathrm{k}^{2}\right]}\end{array}$ \\
\hline$V_{a}$ & & Anode volume $\left[\mathrm{m}^{3}\right]$ \\
\hline$V_{c}$ & 0.005 & Cathode volume $\left[\mathrm{m}^{3}\right]$ \\
\hline$k_{a}$ & 0.01 & Anode conversion factor \\
\hline$k_{c}$ & $7.034 \times 10^{-4}$ & Cathode conversion factor \\
\hline$P_{v s}$ & $7.036 \times 10^{-4}$ & Saturation pressure $[\mathrm{kPa}]$ \\
\hline
\end{tabular}

\subsection{Normal Condition}

In this part, the simulation results for air flow control in PEM fuel cell in normal condition are presented. Normal condition means that system parameters do not have any uncertainties and system would not face any immeasurable disturbance. By considering this assumption, the simulations are done and the results are presented on Figs 6-9. The 
controller was designed to keep the hydrogen and oxygen pressure control around the desired value ( 3 atmosphere), and as it can be seen the proposed adaptive controller and the feedback linearization controller have not significant difference in normal condition. From the Fig.6-9 it is clear that the feedback linearization controller and adaptive controller have better performance in comparison with linear PI controller (e.g. eliminating undershoots and overshoots in the pressure control).

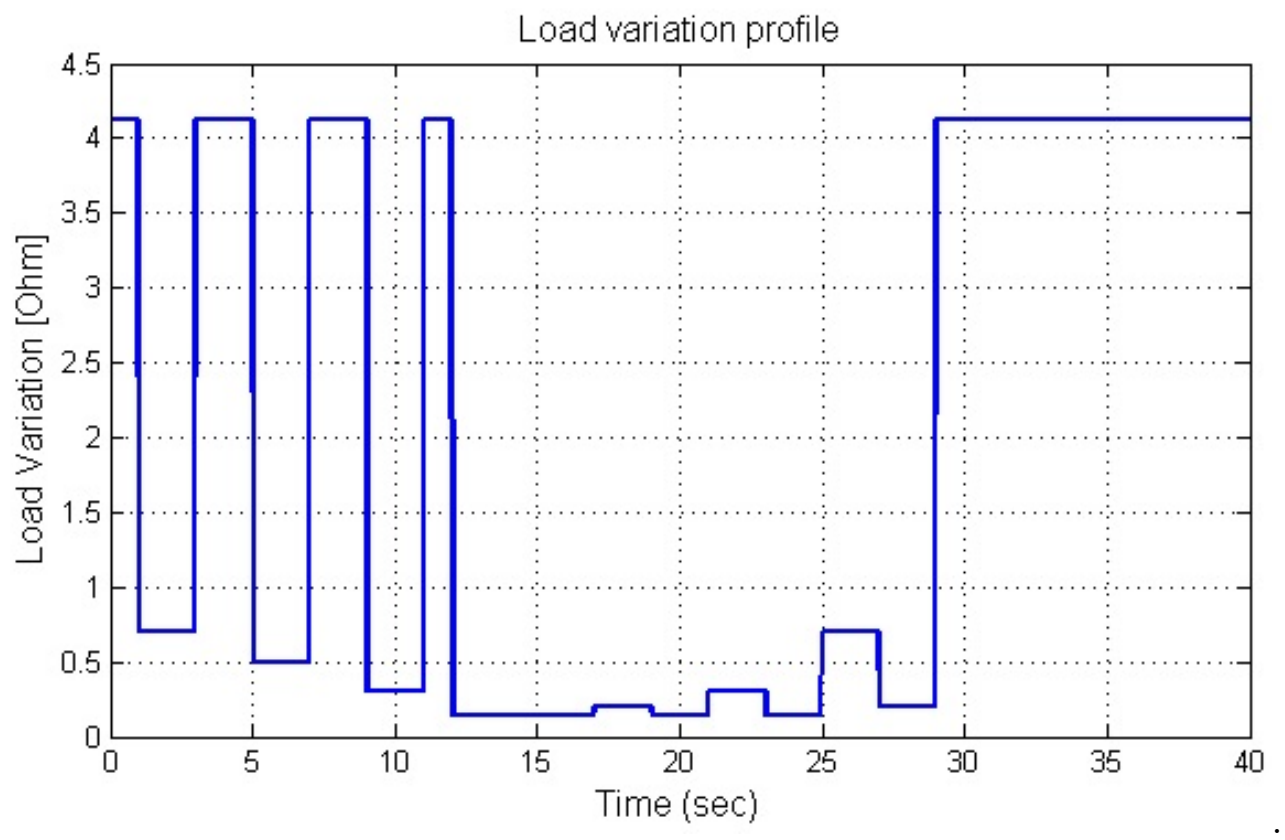

Figure 5: Load variation profile. 


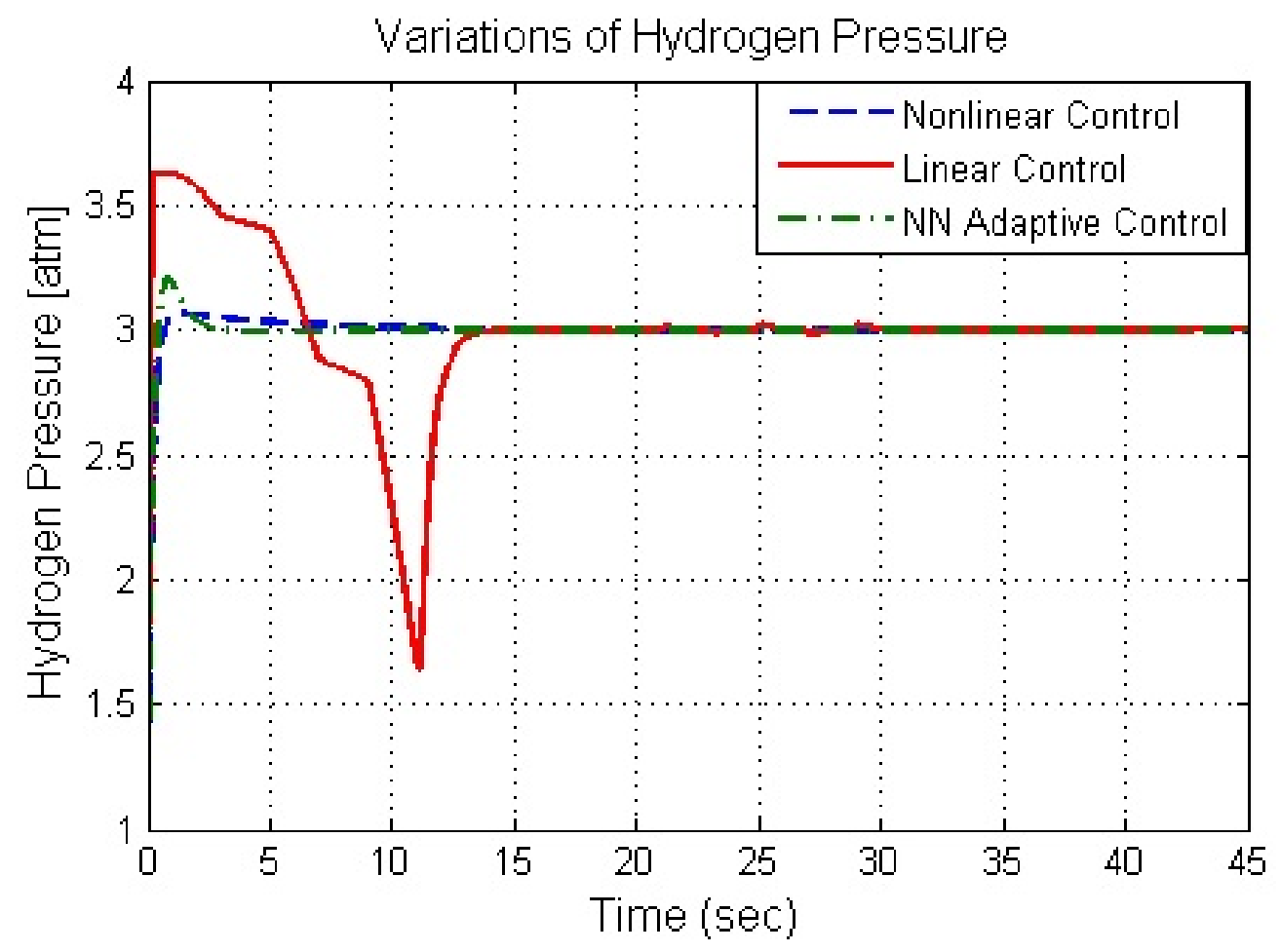

Figure 6: Hydrogen pressure control performance in normal condition.

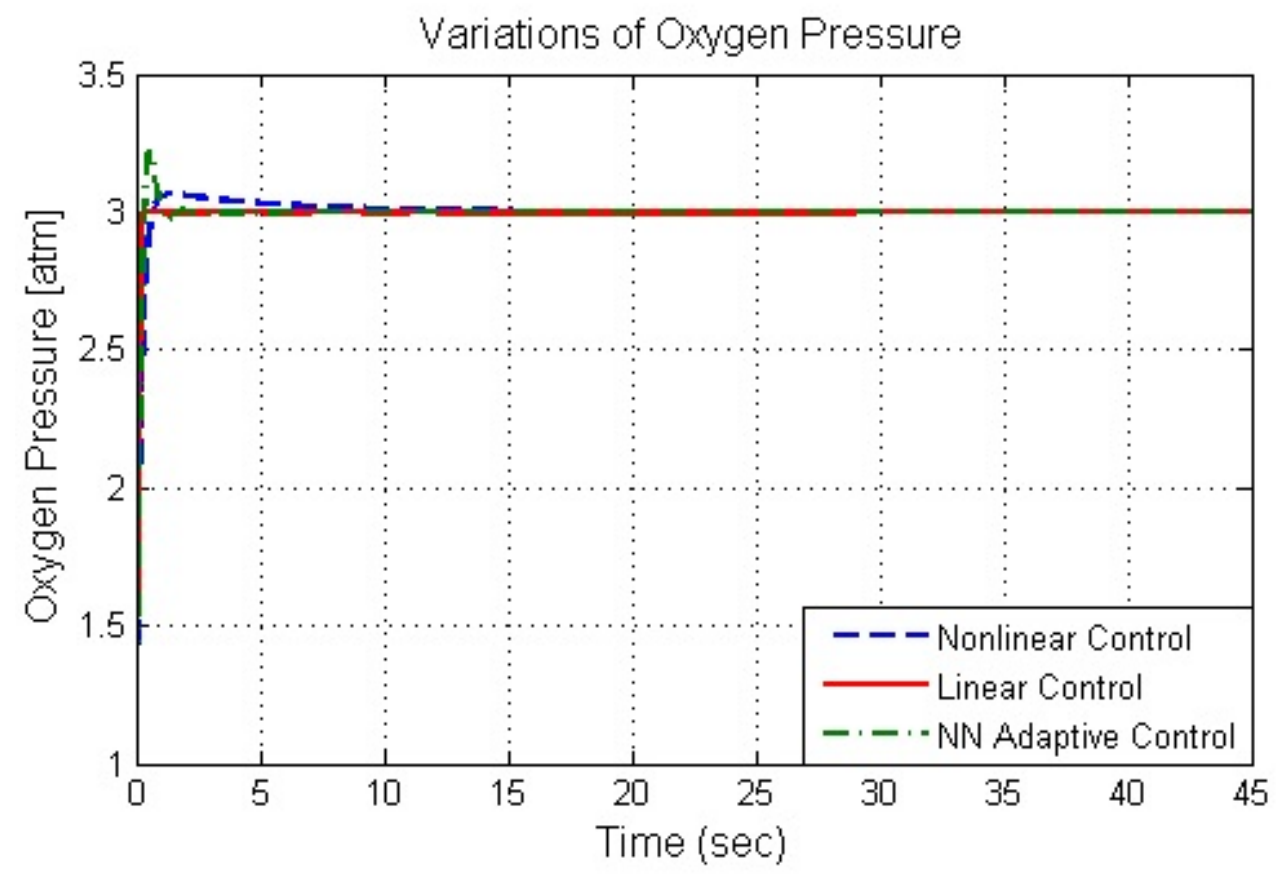

Figure 7: Oxygen pressure control performance in normal condition. 
Fig. 8 and Fig. 9 show the variations of hydrogen and oxygen flow rates, respectively. These flow rates were used as the control inputs of the designed control system, and as it can be seen, the adaptive and feedback linearization controllers use control inputs comparably lesser. Control effort has a direct relation to control input $\left(\int u^{2} d t\right)$, and it is an important factor in designing controllers. Thus, the feedback linearization controller and the proposed adaptive controller are successfully reduced the control effort up to 72 percent in comparison with PI controller. Despite the fact that load control was not the object of this control design, Fig.10 shows that all three controller can handle the load variation in the system.

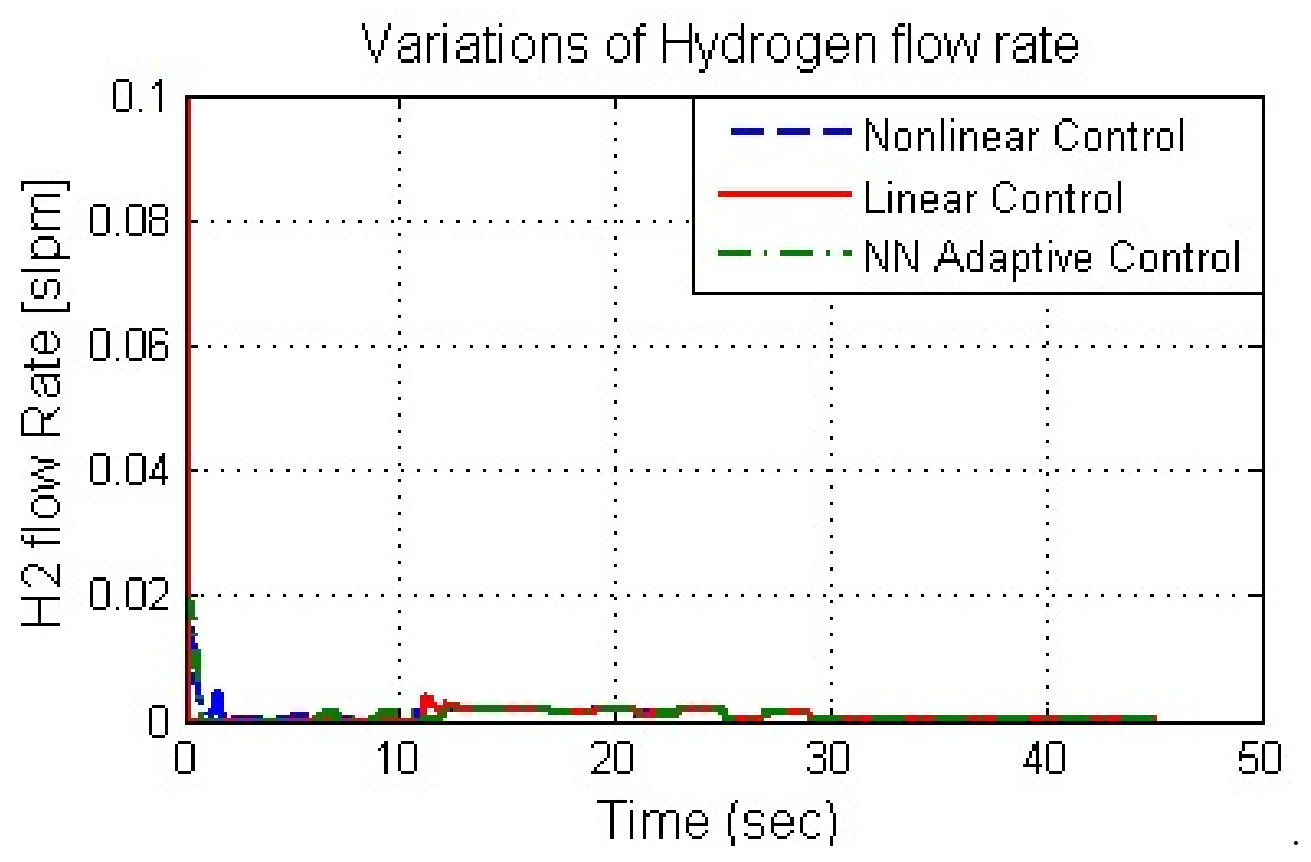

Figure 8: Hydrogen flow rate variations in normal condition. 


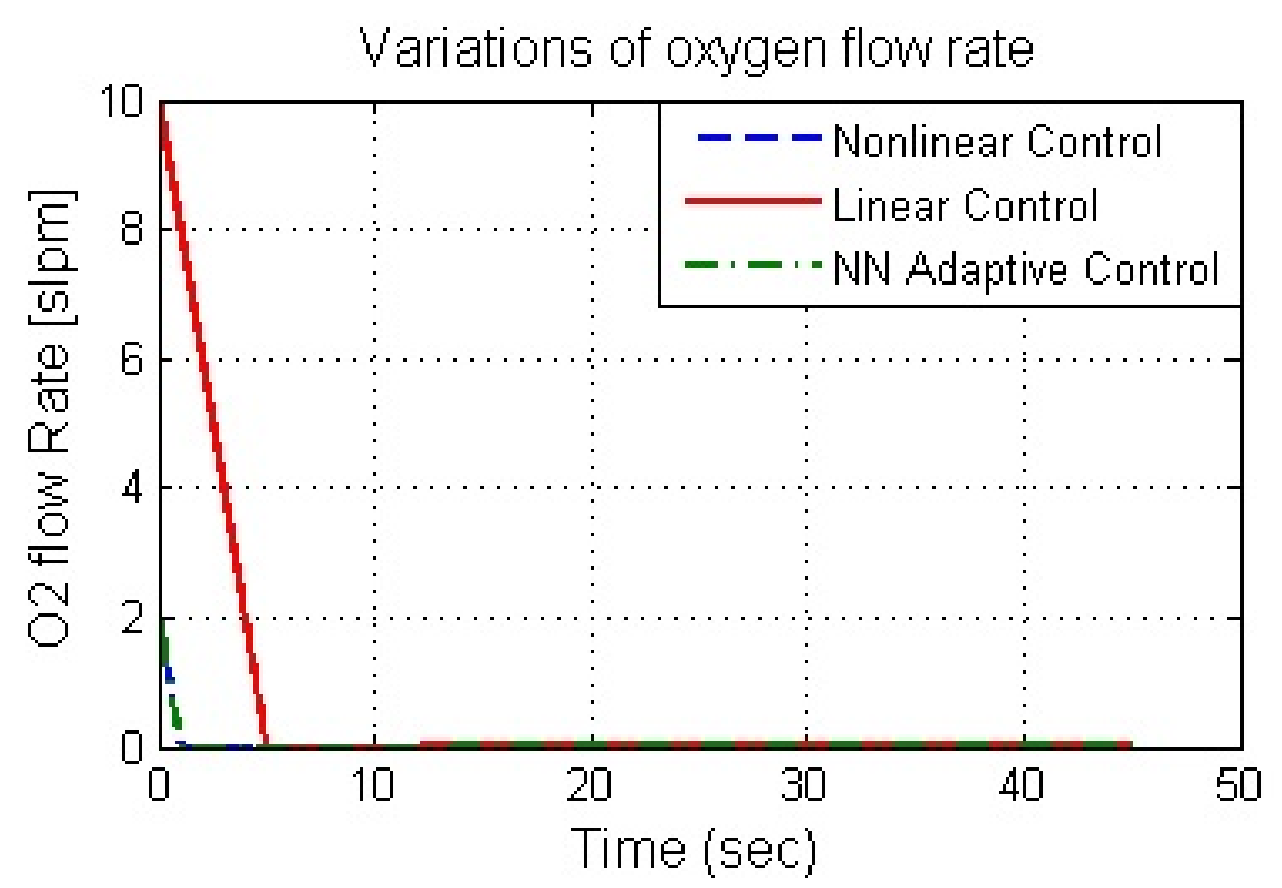

Figure 9: Oxygen flow rate variation in normal condition in normal condition.

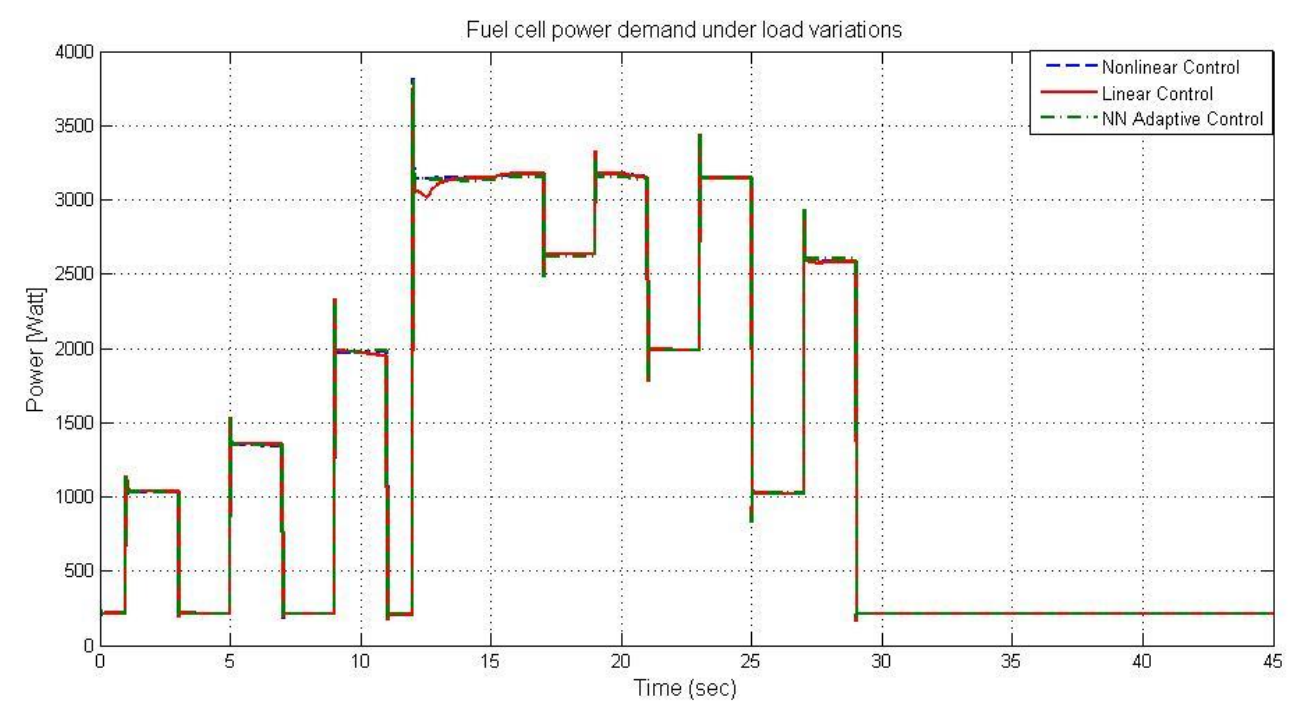

Figure 10: Fuel cell power production under variation of load.

\subsection{Disturbance Rejection}

In this part of simulation we analyzed the system performance in presence of disturbance. We wanted to examine the disturbance rejection ability of our proposed controller.In contrast with the $I_{f c}$ disturbance, this disturbance cannot be measured in the feedback signal, so, in this paper we called it immeasurable disturbance. Fig.11 shows the immeasurable 


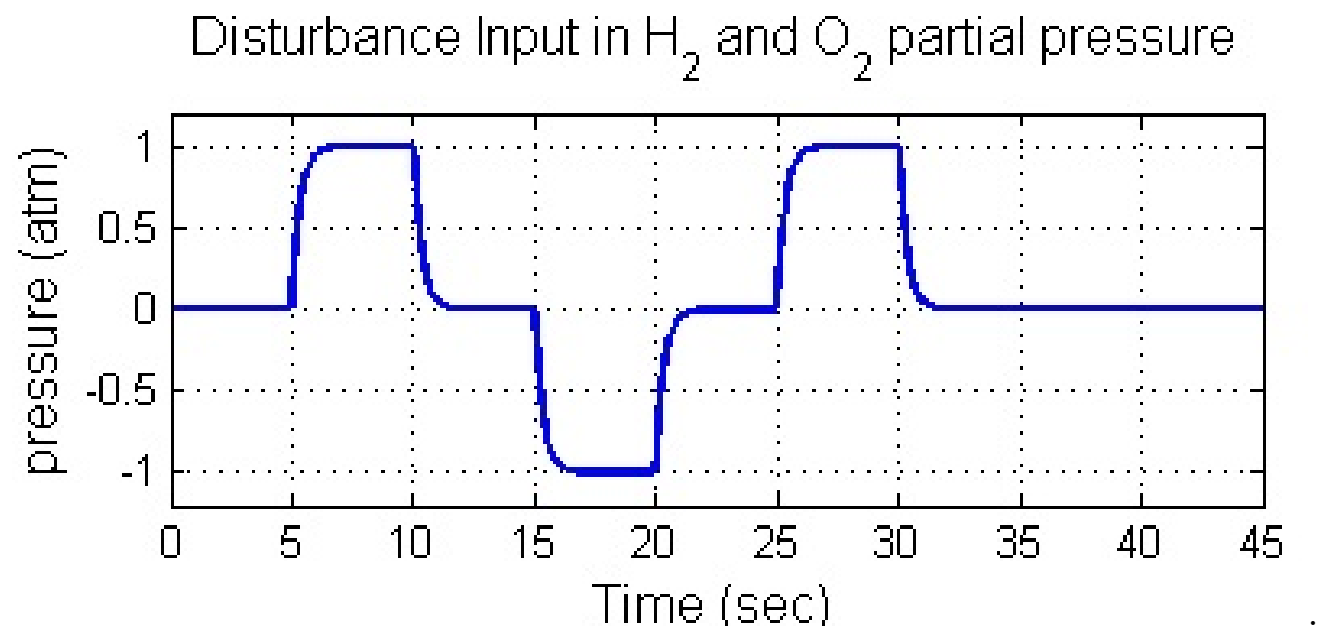

Figure 11: The disturbance inserted to the $\mathrm{O} 2$ an $\mathrm{H} 2$ pressures.

Based on Fig. 12 and Fig. 13 the proposed controller has 6 percent error in presence of large disturbances while the feedback linearization and PI controllers have more than 16 percent error in pressure control. Fig.14 and Fig.15 show the control input of the PEMFC system to control the airflow. In the simulations the actuator model (valve) is modeled, so the simulation results can be achievable in the experimental tests. Fig.16 shows that the NN learning weight matrices converged during the simulation time. These coefficients are tuned during the on-line adaptation. After overcoming the nonlinearities and disturbances in the system, if the learning weight matrices converge to zero, it means that the neural network successfully did its task. In other words, neural network successfully compensated for uncertainties and disturbances in the system. As it can be seen, these matrices converged to zero after the disturbances disappeared. This figure also verifies that the NN can compensate both immeasurable (Fig.11) and measurable $\left(d=I_{f c}\right)$ disturbances effectively. In addition, in comparison with the feedback linearization method, NN helped to decrease the computation time up to 41 percent. 


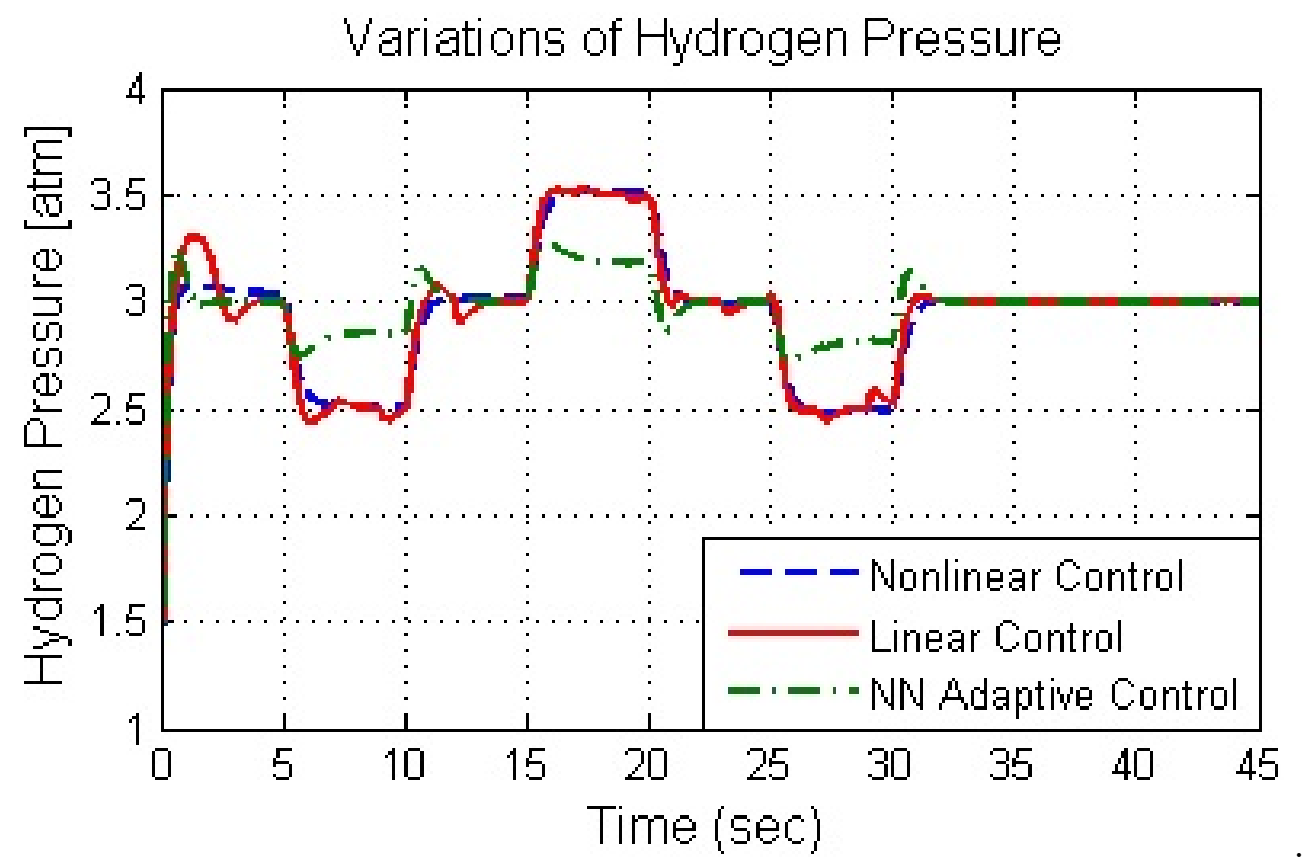

Figure 12: Hydrogen pressure control in presence of immeasurable disturbance.

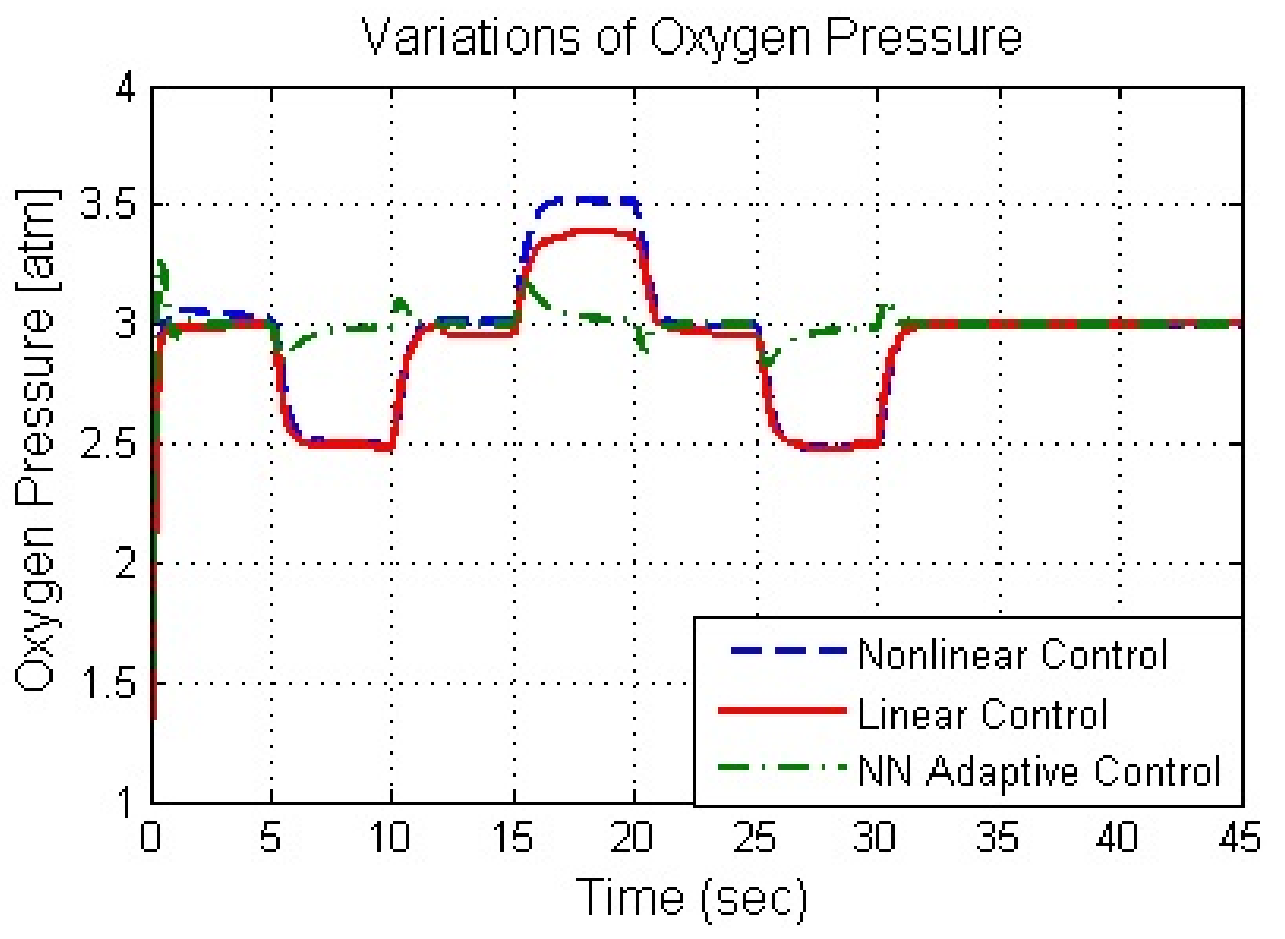

Figure 13: Oxygen pressure control in presence of immeasurable disturbance. 


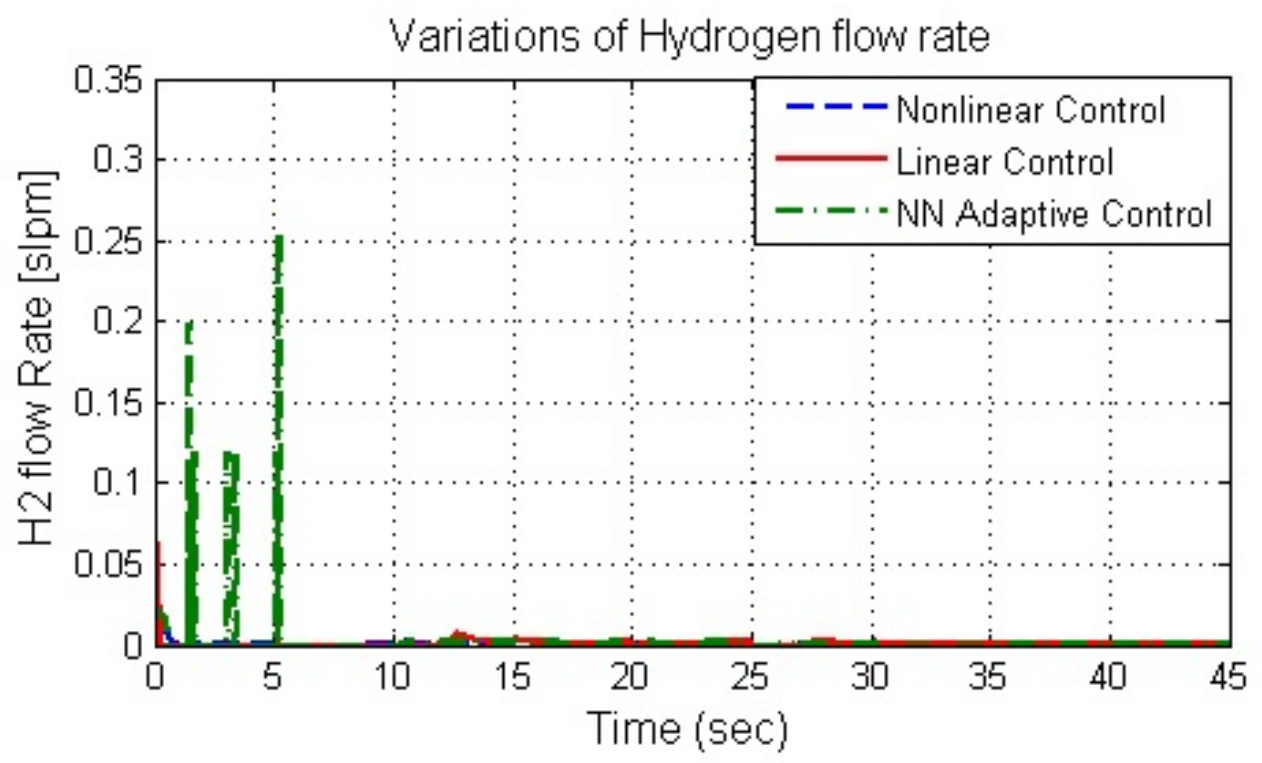

Figure 14: Hydrogen flow rate variations in disturbance condition.

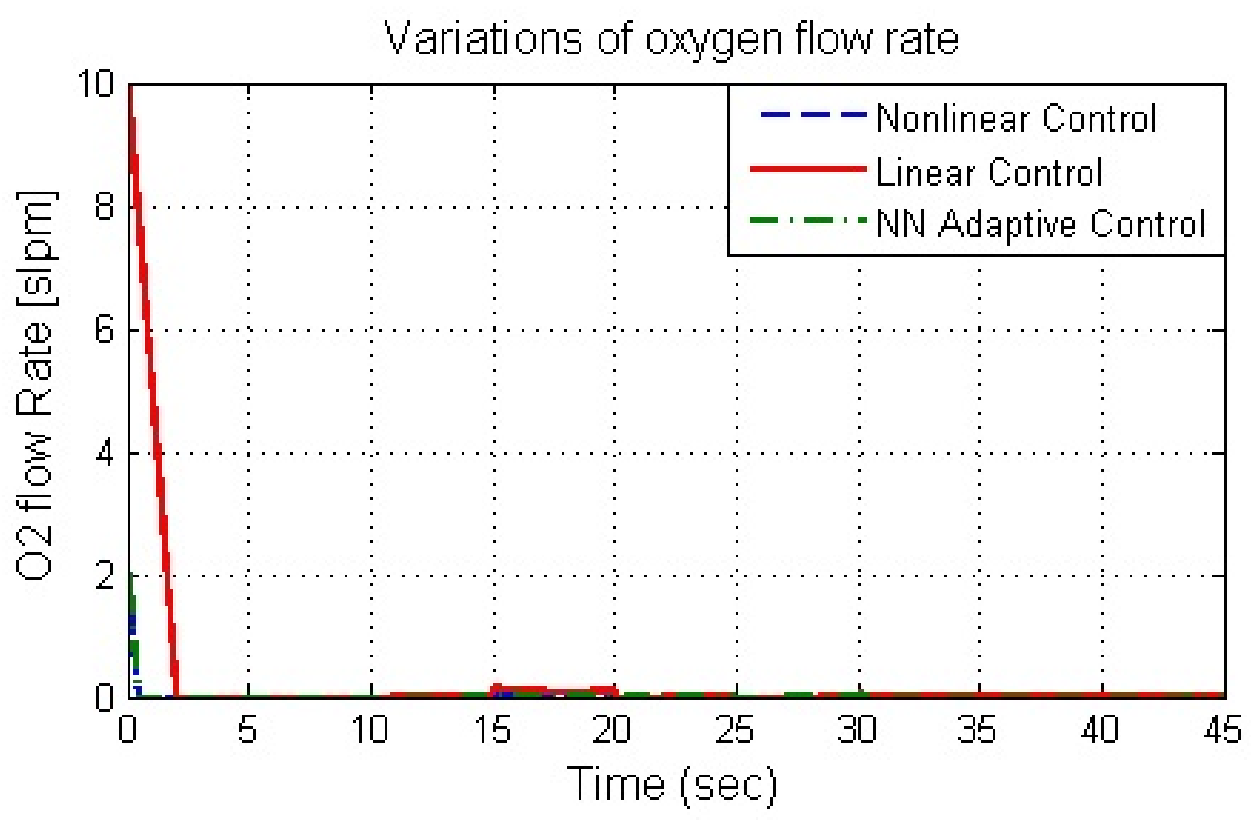

Figure 15: Oxygen flow rate variations in disturbance condition. 


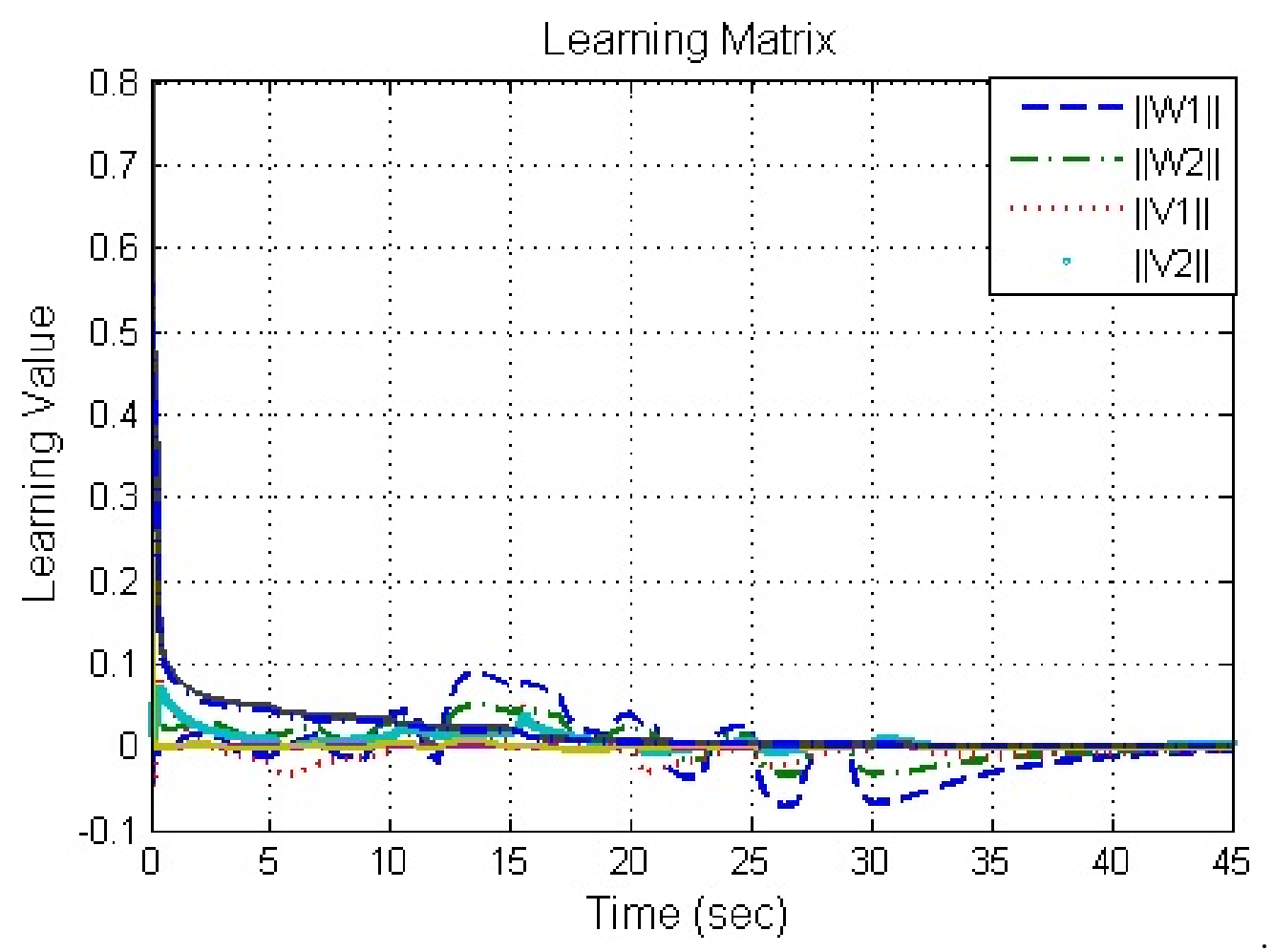

Figure 16: NN weight matrix $(\|W\|,\|V\|)$ versus time.

\subsection{Robustness against uncertainties}

In this section, the robustness of proposed designed is compared with linear PI and feedback linearization controller. Uncertainties which are unknown to system dynamic are inserted to the dynamic system:

$$
\dot{X}=g(x) u+p(x) d+2 \Delta_{f}(x, t)
$$

where $\Delta_{f}$ can be defined as

$$
\Delta_{f}(x, t)=\frac{b_{1}+b_{2} \cos (2 t) x_{1}^{2} x_{3}^{2}}{a_{1}+a_{2} \sin (t)+a_{3} x_{1}^{2}+a_{4} x_{2}^{2}}
$$

where $a_{1}$ and $a_{2}$ are unknown constants which satisfy these inequalities: $a_{1}>a_{2}+1>0$ , $a_{3} \geq 1$ and $a_{4} \geq 1$. Considering mentioned inequalities, the following values are used in the simulation: $a_{1}=2, a_{2}=0.5, a_{3}=a_{4}=1.5, b_{1}=1, b_{2}=-2$. As it was already predicted, Fig.17 and Fig.18 demonstrate that the proposed adaptive controller have better performance than feedback linearization in presence of uncertainties. In Fig.17, it can be seen that the proposed controller decreased the oscillation 20 percent in comparison with the feedback linearization controller. Fig.18 shows that the proposed controller oscillation is 40 percent less than the feedback linearization controller It can be also seen in that linear PI controller is not capable of controlling the PEMFC airflow in presence of parameters uncertainties. Fig. 19 and Fig. 20 show the PEMFC control inputs. These figures show 
that the proposed design has less amplitude and less oscillation in control input actuators, so, it can be concluded that the proposed adaptive controller has better performance even in the control effort.

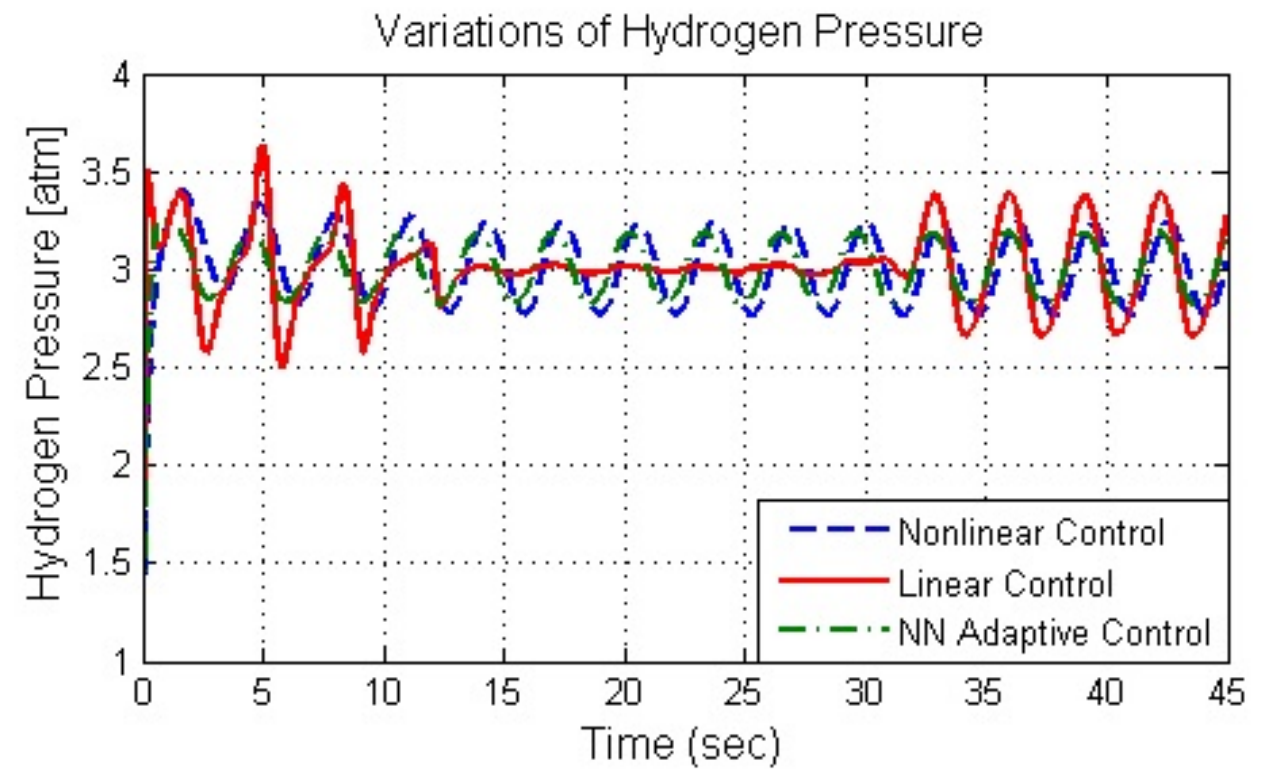

Figure 17: Hydrogen pressure control in a PEM fuel cell system with parameter uncertainties.

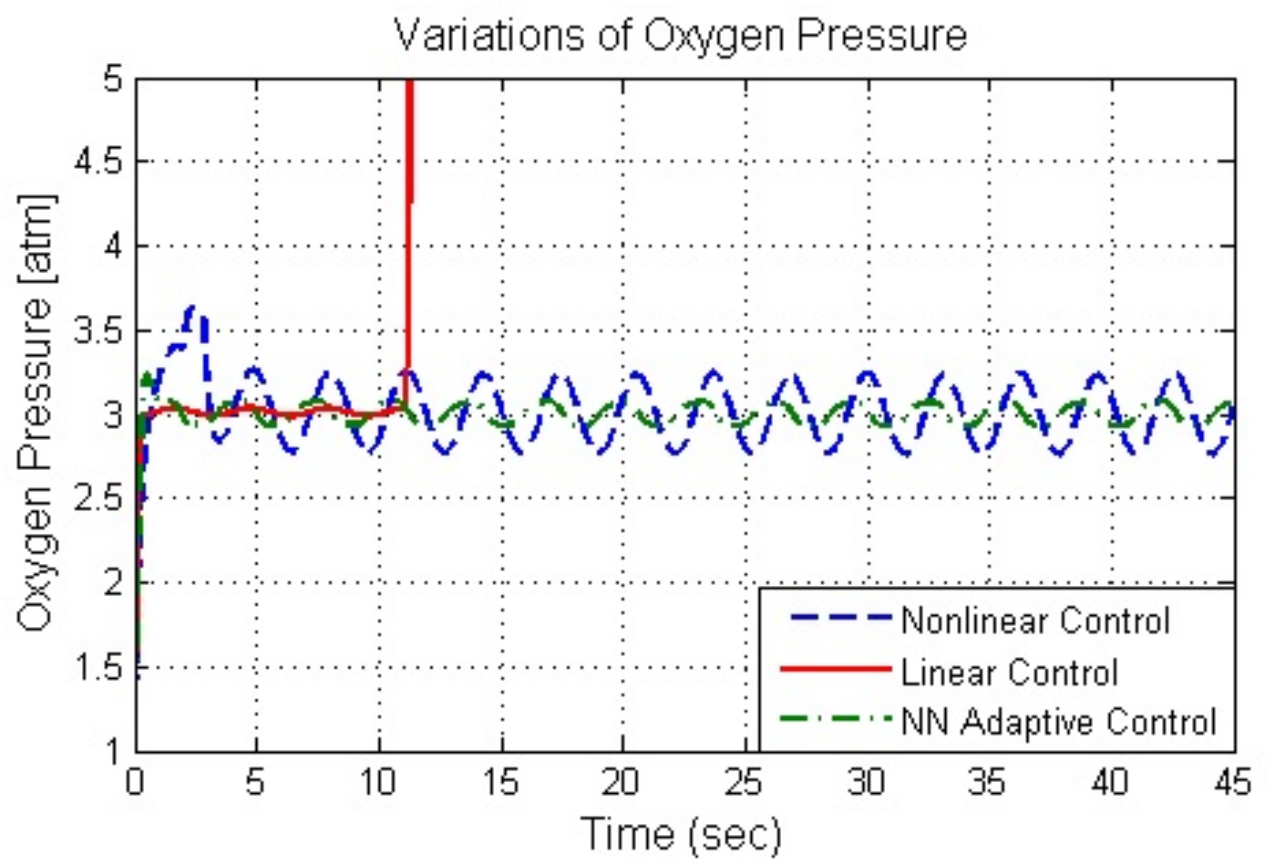

Figure 18: Oxygen pressure control in a PEM fuel cell system with parameter uncertainties. 


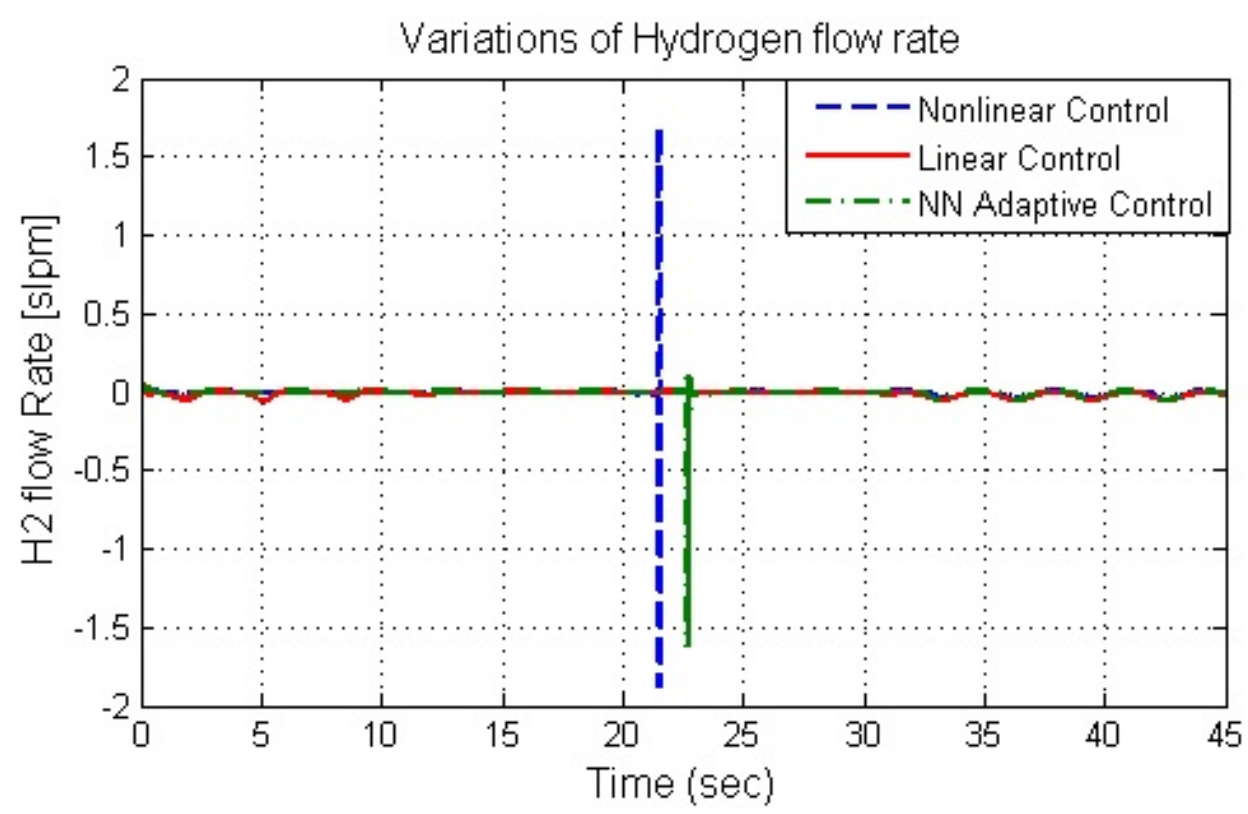

Figure 19: Hydrogen flow rate variations in uncertainties condition.

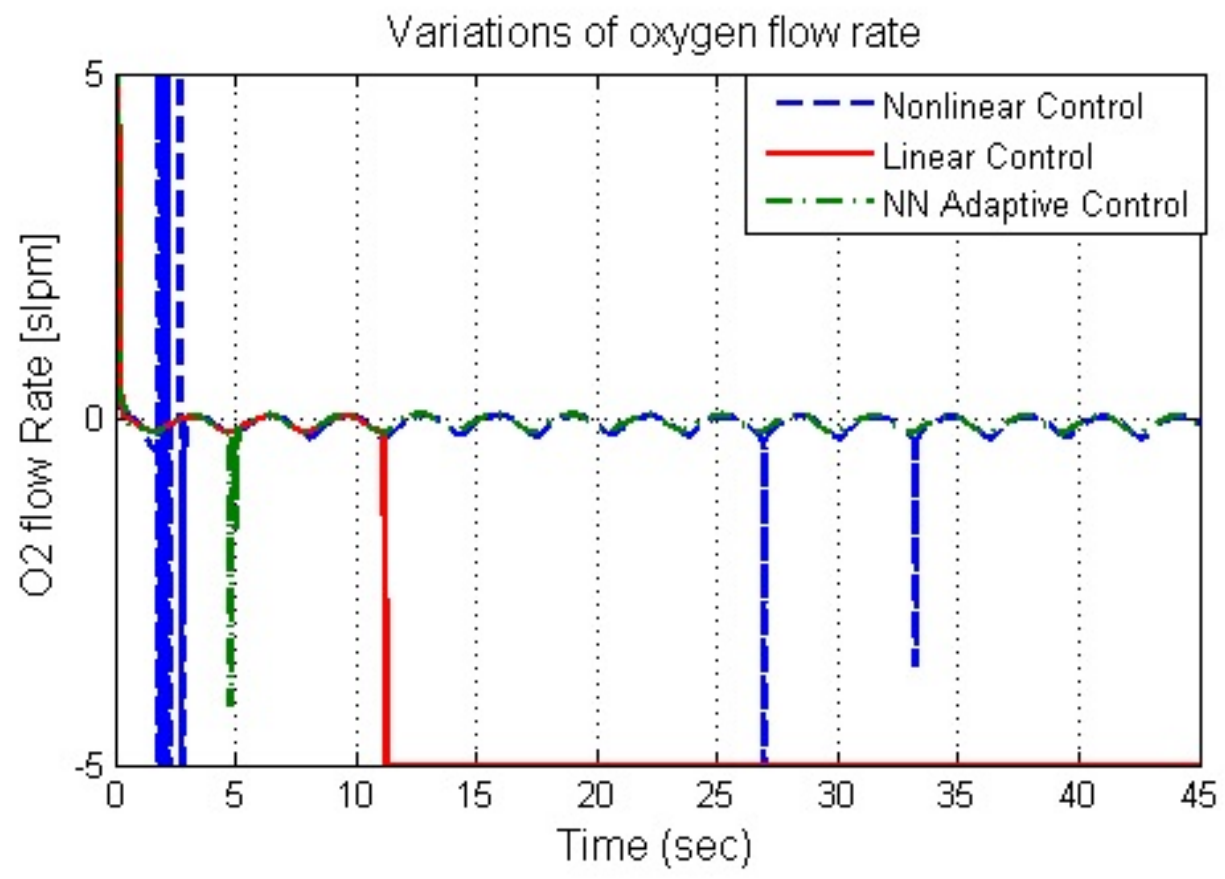

Figure 20: Oxygen flow rate variations in uncertainties condition. 


\subsection{CONCLUSION AND FUTURE WORK}

In this paper, a new approach is introduced to control PEM fuel cells, whereby an adaptive neural network is used to increase the accuracy and robustness of the feedback linearization controller. Consequently, the need to have an accurate dynamic model, which is a requirement for a feedback linearization controller, is diminished. According to simulation results, the introduced neural networks, by adapting its behavior from online learning, successfully compensate for the unknown disturbances and parameter uncertainties on the control performance. This improvement in the control performance prolongs the life of PEMFCs by preventing membrane damage.

In the future, this study will be continued in two directions. First, we will try to demonstrate the advantages of proposed design in experimental tests by implementing the designed controller on a real PEMFC system. Second, we will develop the PEMFC model and control the PEMFC on other system outputs (voltage, current, etc.) at the same time.

\section{Acknowledgement}

This material was based upon work supported in part by the National Science Foundation under the Award No. EPS-0903806 and matching support from the State of Kansas through the Kansas Board of Regents.

\section{References}

[1] J. Purkrushpan, A. G. Stefanopoulou, and H. Peng, Control of fuel cell breathing, IEEE Control Syst. Mag., Vol. 24, No. 2, pp. 30-46, Apr. 2004.

[2] Woon ki Na and Bei Gou, "Feedback linearization based nonlinear control for PEM fuel cells," IEEE Transaction on Energy Conversion, Vol. 23, No. 1, pp. 179-190, March 2008.

[3] M. J. Khan and M. T. Labal, "Dynamic modeling and simulation of a fuel cell generator," Fuel Cells, Vol. 5, No. 1, pp. 97-104, 2005.

[4] C. Wang, M. H. Nehrir, and S. R. Shaw, "Dynamic model and model validation for PEM fuel cells using electrical circuits," IEEE Trans. Energy Convers., Vol. 20, No. 2, pp. 442-451, June 2005.

[5] L. Y. Chiu, B. Diong, and R. S. Gemmen, "An improved small-signal mode of the dynamic behavior of PEM fuel cells," IEEE Trans. Ind. Appl., Vol. 40, No. 4, pp. 970-977, July/Aug. 2004.

[6] B. Larbi, , W. Alimi, R.Chouikh, A. Guizani, "Effect of porosity and pressure on the PEM fuel cell performance", International Journal of Hydrogen Energy, Vol. 38, Issue 20, PP. 8542-85499, July 2013.

[7] Faysal Tiss, , Ridha Chouikh, Amenallah Guizani, "Dynamic modeling of a PEM fuel cell with temperature effects", International Journal of Hydrogen Energy, Vol. 38, Issue 20, pp: 8532-8541, 9 July 2013.

[8] Restrepo, Carlos, Tine Konjedic, Alejandro Garces, Javier Calvente, and Roberto Giral. "Identification of a proton-exchange membrane fuel cell's model parameters by means of an evolution strategy." Industrial Informatics, IEEE Transactions on 11, no. 2 (2015): 548-559.

[9] da Costa Lopes, Francisco, Edson H. Watanabe, and Luis Guilherme B. Rolim. "A Control-Oriented Model of a PEM Fuel Cell Stack Based on NARX and NOE Neural Networks." Industrial Electronics, IEEE Transactions on 62, no. 8 (2015): 5155-5163.

[10] P. E. M. Almeida and M. Godoy, "Neural optimal control of PEM fuel cells with parametric CMAC network," IEEE Trans. Ind. Appl., Vol. 41, No. 1, pp. 237-245, Jan./Feb. 2005.

[11] A. Rezazadeh, A. Askarzadeh, M. Sedighizadeh, "Adaptive inverse control of proton exchange membrane fuel cell using RBF neural network," International Journal of Electrochemical Science, Vol. 6, pp. $3105-3117,2011$. 
[12] Alin C. Fărcaş, Petru Dobra, "Adaptive control of membrane conductivity of PEM fuel cell," Journal of Procedia Technology, Vol. 12, pp. 42-49, 2014.

[13] A. Abaspour, N.T.Parsa, M. Sadeghi, "A new feedback linearization-NSGA-II based control design for PEM fuel cell," International Journal of Computer Applications, Vol. 97, No. 10, pp. 17044-7354, July 2014.

[14] H. Beirami , A. Zargar Shabestari, M.Mahdi Zerafat, "Optimal pid plus fuzzy controller design for a pem fuel cell air feed system using the self-adaptive differential evolution algorithm", ", International Journal of Hydrogen Energy, Vol.40, Issue 30, PP. 9422-9434, 10 August 2015.

[15] Kai Ou, Ya-Xiong Wang, Zhen-Zhe Li, Yun-De shen, dong-ji xuan, "Feedforward fuzzy-pid control for air flow regulation of pem fuel cell system", International Journal of Hydrogen Energy, Vol. 40, Issue 35, pp: 11686-11695, 21 September 2015.

[16] Benchouia, Nedjem Eddine, Abdallah Derghal, Bouziane Mahmah, Belgacem Madi, Lakhdar Khochemane, and Elias Hadjadj Aoul. "An adaptive fuzzy logic controller (AFLC) for PEMFC fuel cell." International Journal of Hydrogen Energy 40, no. 39 (2015): 13806-13819.

[17] Wang, Fu-Cheng, and Yi-Fu Guo. "Robustness analyses of PEMFC systems on the production line." International Journal of Hydrogen Energy 40, no. 4 (2015): 1959-1966.

[18] Li, Zhongliang, Rachid Outbib, Stefan Giurgea, and Daniel Hissel. "Diagnosis for PEMFC systems: a data-driven approach with the capabilities of online adaptation and novel fault detection." Industrial Electronics, IEEE Transactions on 62, no. 8 (2015): 5164-5174.

[19] Lebreton, Carole, Michel Benne, Cédric Damour, Nadia Yousfi-Steiner, Brigitte Grondin-Perez, Daniel Hissel, and Jean-Pierre Chabriat. "Fault Tolerant Control Strategy applied to PEMFC water management." International Journal of Hydrogen Energy 40, no. 33 (2015): 10636-10646.

[20] J. Larminie and A. Dicks, Fuel Cell Systems Explained. New York: Wiley, 2002.

[21] F. Barbir, "PEM Fuel Cells: Theory and Practice". London, U.K. Elsevier, 2005.

[22] A. Isidori, Nonlinear Control Systems. 3rd ed. London, U.K.: Springer Verlag, 1995.

[23] J. J. E. Slotine and W. Li, Applied Nonlinear Control. Englewood Cliffs, NJ: Prentice-Hall, 1991.

[24] M. A. Henson and D. E. Seborg, "Critique of exact linearization strategies for process control," J. Process Control, Vol. 1, pp. 122-139, May 1991.

[25] Nakwan Kim," Improved methods in neural network based adaptive output feedback control, with application to flight control," School of Aerospace Engineering, Georgia Institute of Technology, Nov. 2003.

[26] Taeyoung Lee, Youdan Kim, "Nonlinear adaptive flight control using backstepping and neural networks Controller," Journal of Guidance, Control, and Dynamics, Vol. 24, No. 4, July-August 2001.

[27] S. Lee, C.Ha, B.S.Kim, "Adaptive nonlinear control system design for helicopter robust command augmentation," Aerospace Science and Technology, Vol. 9 , pp. 241-251, 2005.

[28] A. Abaspour, S.H. Sadati, M. Sadeghi, "Nonlinear optimized adaptive trajectory control of helicopter", Control Theory and Technology, Vol. 13, Issue 4, pp 297-310, November 2015. 